(C) 2013 Institute of Physics and Engineering in Medicine

This document is published in: J. Cal-González, J.L. Herraiz, S. España, P.M.G. Corzo, J.J. Vaquero, M. Desco and J.M. Udias, (2013). Positron range estimations with PeneloPET. In: Physics in Medicine and Biology, (August 2013), vol. 58, n. 15, pp. 5127-5152.

DOI: http://dx.doi.org/10.1088/0031-9155/58/15/5127 


\title{
Positron range estimations with PeneloPET
}

\author{
J Cal-González ${ }^{1}$, J L Herraiz ${ }^{2}$, S España ${ }^{3}$, P M G Corzo ${ }^{1}$, J J Vaquero ${ }^{4}$, \\ $\mathbf{M}_{\text {Desco }^{4,5}}$ and $\mathbf{J} \mathbf{M}$ Udias $^{1}$ \\ ${ }^{1}$ Grupo de Física Nuclear, Departamento de Física Atómica, Molecular y Nuclear, \\ Universidad Complutense de Madrid, CEI Moncloa, Spain \\ 2 Madrid-MIT M+Vision Consortium, Research Lab of Electronics, MIT, Cambridge, MA, USA \\ ${ }^{3}$ Medical Image and Signal Processing Group, Department of Electronics and Information \\ Systems, Ghent University Hospital, Belgium \\ ${ }^{4}$ Departamento de Bioingeniería e Ingeniería Aeroespacial, Universidad Carlos III de Madrid, \\ Spain \\ ${ }^{5}$ Unidad de Medicina y Cirugía Experimental, Hospital General Universitario Gregorio \\ Marañón. CIBERSAM, Madrid, Spain
}

\begin{abstract}
Technical advances towards high resolution PET imaging try to overcome the inherent physical limitations to spatial resolution. Positrons travel in tissue until they annihilate into the two gamma photons detected. This range is the main detector-independent contribution to PET imaging blurring. To a large extent, it can be remedied during image reconstruction if accurate estimates of positron range are available. However, the existing estimates differ, and the comparison with the scarce experimental data available is not conclusive. In this work we present positron annihilation distributions obtained from Monte Carlo simulations with the PeneloPET simulation toolkit, for several common PET isotopes $\left({ }^{18} \mathrm{~F},{ }^{11} \mathrm{C},{ }^{13} \mathrm{~N},{ }^{15} \mathrm{O},{ }^{68} \mathrm{Ga}\right.$ and $\left.{ }^{82} \mathrm{Rb}\right)$ in different biological media (cortical bone, soft bone, skin, muscle striated, brain, water, adipose tissue and lung). We compare PeneloPET simulations against experimental data and other simulation results available in the literature. To this end the different positron range representations employed in the literature are related to each other by means of a new parameterization for positron range profiles. Our results are generally consistent with experiments and with most simulations previously reported with differences of less than $20 \%$ in the mean and maximum range values. From these results, we conclude that better experimental measurements are needed, especially to disentangle the effect of positronium formation in positron range. Finally, with the aid of PeneloPET, we confirm that scaling approaches can be used to obtain universal, material and isotope independent, positron range profiles, which would considerably simplify range correction.
\end{abstract}




\section{Introduction}

One of the most important sources of resolution degradation in PET imaging is the positron range, particularly for certain radioisotopes and materials. The degree of image blurring depends on the positron kinetic energy, since larger energies lead to longer paths before annihilation. Positron range also depends on the surrounding tissue type in such a way that in those tissues with lower electron density positron ranges are larger. The blurring effect of the positron range may be described as a contribution to the system point spread function (PSF) (Derenzo 1986, Haber et al 1990). Recent studies have proposed the use of space-variant PSF functions (Rapisarda et al 2010) to obtain optimal range-corrected PET images (Bai et al 2003, 2005, Fu and Qi 2010, Cal-González et al 2011). In order to develop accurate positron range correction techniques it is essential to properly characterize the positron range, both with simulation and experimental studies. Positron range correction in image reconstruction is of particular importance when dealing with small-animal PET studies or when radioisotopes with large positron range are involved.

Nowadays, the radioisotope more commonly used in PET is ${ }^{18} \mathrm{~F}$, especially in the form of $\left[{ }^{18} \mathrm{~F}\right]-\mathrm{FDG}$, for exploring glucose metabolism in tumours and some cerebral or cardiac diseases. Other radioisotopes with shorter half-lives such as ${ }^{11} \mathrm{C}(20.1 \mathrm{~min}),{ }^{15} \mathrm{O}(2.07 \mathrm{~min})$ or ${ }^{13} \mathrm{~N}$ (10 min) can only be used in centres with a cyclotron facility. Other non-cyclotron-based radioisotopes such as ${ }^{68} \mathrm{Ga}(68.1 \mathrm{~min})$ and ${ }^{82} \mathrm{Rb}(1.3 \mathrm{~min})$ have also been included in our study because they have increasing use in medical diagnosis (Hoffend et al 2005, Breeman and Verbruggen 2007, Yoshinaga et al 2006).

Early experimental efforts (Phelps et al 1975, Cho et al 1975, Hoffman et al 1976) to measure positron range in water for medically important positron emitters were of limited accuracy because the resolution of the detector was comparable to the positron range. Derenzo (1979) measured annihilation point spread distributions in low-density polyurethane foam, where positron range is significantly larger. Positron range distribution data were deconvoluted, fitted to an empirical function, and finally scaled to water equivalent values using range-energy relations derived for electrons (Katz and Penfold 1952). There are several potential difficulties inherent to this approach (Levin and Hoffman 1999), such as the need to extrapolate range results from polyurethane to water or the possible loss of information due to the deconvolution. Palmer and Brownell (1992) evaluated annihilation density distributions for certain positron emitters through calculations based on beta-decay energy spectra combined with an empirical range formula, assuming that positrons behave diffusively. More recently, several authors have studied the reduction of positron range in presence of a magnetic field (Wirrwar et al 1997, Herzog et al 2010). According to Soultadinis et al 2011 and Kraus et al 2012, positron range is unaffected or just slightly enlarged along the direction of the magnetic field, thus producing an anisotropic distribution of annihilations.

The difficulties involved in the experimental measurements of positron range support the widespread use of Monte Carlo simulations (Levin and Hoffman 1999, Harrison et al 1999, Champion and Le Loirec 2006, 2007, Le Loirec and Champion 2007a, 2007b, 2007c). Most of these studies use water as a reference medium for positron interaction. The exclusive use of water-based data may be misleading since positron range depends on density and atomic composition of the tissue (Sánchez-Crespo et al 2004, Alessio and MacDonald 2008, Lehnert et al 2011, Jødal et al 2012). An accurate assessment of the consequences of positron range in PET imaging requires a specific model for each tissue type.

The correction of positron range effects in PET image reconstruction is becoming mandatory in the light of the increasing use of high-energy positron emitters in clinical PET imaging, and the excellent spatial resolution of modern PET scanners, even sub-millimetric for 
some small-animal systems. Some studies have addressed the question of how accurately the positron range should be known to obtain good range-corrected PET images (Cal-González et al 2011). In these studies, phantoms were simulated with a given positron range profile. These simulated data were then reconstructed with an iterative reconstruction method which incorporates a model of the PET scanner and the effect of positron range. The average positron range for the profiles employed during reconstruction was varied. Actually, the shape of the profiles was just rescaled in the spatial coordinate to obtain a change in the average positron range. The effect on the reconstructed images was assessed. It was found that differences of about $10 \%$ in the average positron range of the profiles employed to correct the reconstruction with respect to the profiles employed during the simulation of the acquisition, translate into detectable changes in image quality. This raises the question of whether positron range profiles are known with such accuracy for the radioisotopes and materials of current interest for PET imaging. A first review of the available positron range data in the literature, either from simulations or from experiments, reveals discrepancies larger than $20 \%$ in many cases (Levin and Hoffman 1999, Champion and Le Loirec 2007). However, as pointed by Jødal et al 2012, different authors chose different ways of representing range profiles, which precludes a straightforward comparison.

As suggested by Jødal et al (2012), positron range profiles can be scaled to obtain universal profiles independent of the density of materials and energy of the emitter. This approach may facilitate the implementation of fast algorithms to correct images for positron range during reconstruction, if these scaling approaches are accurate at the said 10\%, or lower, in the average positron range.

Thus, the goals of this paper are the following:

- To obtain positron range distributions for common PET isotopes in various human tissues, employing a simulation tool, PeneloPET.

- To develop a general framework and a tool to convert from any of the multiple ways employed in the literature to show positron range into another. Using this framework, we make a general comparison of PeneloPET predictions for positron range for the available experimental data and also for simulations from other authors.

- To confirm the possibility of employing material-independent scaled positron range distributions, as pointed out by Jødal et al (2012). We employ a semiempirical parameterization of positron range, and verify the validity of the material and isotope scaling approach with PeneloPET, for different combinations of radioisotopes and materials.

\section{Monte Carlo simulation}

There are several Monte Carlo codes able to simulate the transport of radiation through matter, e.g. GEANT4 (Agostinelli 2003, Allison et al 2006), MCNP (X-5 Monte Carlo Team 2003), EGS5 (Hirayama et al 2005) and PENELOPE (Baró et al 1995). We have chosen PENELOPE (2008 version, with the standard simulation parameters, as recommended in Salvat et al (2008)) to simulate positron trajectories. PENELOPE describes the transport in matter of electrons, positrons and photons with energies from a few hundred eV to $1 \mathrm{GeV}$. PENELOPE is then less generally aimed as GEANT4, but it suits well PET needs, it is fast and robust, and it is being extensively used for medical physics applications, particularly for dosimetry and radiotherapy (Sempau and Andreo 2006, Panettieri et al 2007). Many Monte Carlo codes employ multiple dispersion models for the transport of electrons and positrons, which allow for the global simulation of a large number of interactions in a condensed form. This condensed history 
methodology may cause a loss of accuracy when facing media boundary. PENELOPE, on the other side, considers in detail the transport of electrons and positrons, interaction by interaction, which makes it particularly suited to simulate positron range, eventually even across media boundaries. PENELOPE has been shown to produce good results up to distances below one millimetre (Muñoz et al 2005). Positron interactions and subsequent annihilation simulated with PENELOPE have been included in PeneloPET (España et al 2009). PeneloPET is built on top of PENELOPE and provides a simple framework to simulate PET scanners. PeneloPET is freely available and can be obtained under request (see http://nuclear.fis.ucm.es/penelopet).

\subsection{Energy spectra}

When a positron is generated in a decay process, it has a kinetic energy that depends on the energy shared with the neutrino created in the same process. This yields a continuum energy spectrum distribution whose theoretical grounds are well-known, both for allowed or super allowed transitions as well as for forbidden decays, and the energy spectrum can be computed with accuracy (Gove and Martin 1971), including the Coulomb correction factor (Wu and Moskowski 1966). For allowed decays, the theoretical energy distribution (Fermi 1934) has the form:

$$
N(E) \mathrm{d} E \equiv g F(Z, E) p E\left(E_{\max }-E^{2}\right) \mathrm{d} E
$$

Where $g$ is a constant, $E$ is the positron kinetic energy and $p$ is the corresponding momentum, in units of $m c^{2}$ and $m c$, respectively. $E_{\max }$ corresponds to the maximum kinetic positron energy. Table 1 reports $E_{\max }$ for the isotopes considered, along with the decay mode, the parent halflife, the mean kinetic positron energy $E_{\text {mean }}$, and the intensity for each positron and gamma ray emitted in the decay. Note that in this table an intensity of $100 \%$ corresponds to the emission of one particle (photon or positron in $\beta^{+}$decay) per decay. In the case of annihilation photons, one can have up to two photons emitted per decay, that is, $200 \%$ intensity. $F(Z, E)$ is the Fermi function which takes into account the effect of the nuclear Coulomb field in the electron or positron emitted. In positron range simulation, this latter Coulomb correction factor is often either neglected or just treated within a non-relativistic approach (see, for instance, Levin and Hoffman 1999). PeneloPET uses the exact expression for the Coulomb correction factor (Fermi 1934), which, albeit relatively close to one for light nuclei and positron emission, it may significantly distort the beta spectrum for medium to heavy nuclei, particularly in the case of electron emission (España et al 2009). The energy spectra simulated for the radioisotopes of interest are shown in figure 1.

\subsection{Electron and positron interactions and transport mechanism}

The possible interactions of electrons and positrons within a medium of interest for positron range estimates are: elastic scattering, inelastic collisions and bremsstrahlung emission; positrons can also undergo annihilation, either in flight or at rest. The dominant energy loss mechanisms for electrons and positrons with intermediate and low energies are inelastic collisions. For a detailed description of the way PENELOPE deals with inelastic collisions of electrons and positrons, see Liljequist 1987, Salvat and Fernandez-Varea 1992 and Fernandez-Varea et al 1993. For the simulation of electron and positron transport mechanisms, PENELOPE implements a 'mixed' simulation scheme (Andreo and Brahme 1984), which combines the detailed simulation of hard events with condensed simulation of soft events (Fernandez-Varea et al 1993, Baró et al 1995, Bielajew and Salvat 2001). 
Table 1. Decay mode, half-life, maximum and mean kinetic energies ( $E_{\max }$ and $E_{\text {mean }}$ respectively), and intensity of the annihilation photons emitted by the radioisotope considered in his work (NNDC 2011).

\begin{tabular}{|c|c|c|c|c|c|c|}
\hline Isotopes & ${ }^{18} \mathrm{~F}$ & ${ }^{11} \mathrm{C}$ & ${ }^{13} \mathrm{~N}$ & ${ }^{15} \mathrm{O}$ & ${ }^{68} \mathrm{Ga}$ & ${ }^{82} \mathrm{Rb}$ \\
\hline $\begin{array}{l}\text { Decay mode } \\
\text { (Branching ratio) }\end{array}$ & $\begin{array}{l}\beta^{+} \\
(96.7 \%)\end{array}$ & $\begin{array}{l}\beta^{+} \\
(99.8 \%)\end{array}$ & $\begin{array}{l}\beta^{+} \\
(99.8 \%)\end{array}$ & $\begin{array}{l}\beta^{+} \\
(99.9 \%)\end{array}$ & $\begin{array}{l}\beta^{+} \\
(89.1 \%)\end{array}$ & $\begin{array}{l}\beta^{+} \\
(95.4 \%)\end{array}$ \\
\hline Parent nucleus & ${ }^{18} \mathrm{~F}$ & ${ }^{11} \mathrm{C}$ & ${ }^{13} \mathrm{~N}$ & ${ }^{15} \mathrm{O}$ & $\begin{array}{l}{ }^{68} \mathrm{Ga} \\
\left(\text { Generator: }{ }^{68} \mathrm{Ge}\right)\end{array}$ & $\begin{array}{l}{ }^{82} \mathrm{Rb} \\
\text { (Generator: }{ }^{82} \mathrm{Sr} \text { ) }\end{array}$ \\
\hline Parent $\mathbf{J}^{\pi}$ & $1^{+}$ & $3 / 2^{-}$ & $1 / 2^{-}$ & $1 / 2^{-}$ & $1^{+}$ & $1^{+}$ \\
\hline Daughter $\mathbf{J}^{\pi}$ & $0^{+}$ & $3 / 2^{-}$ & $1 / 2^{-}$ & $1 / 2^{-}$ & $0^{+}, 2^{+}$ & $0^{+}, 2^{+}$ \\
\hline Type of transition & Allowed & Allowed & Allowed & Allowed & Allowed & Allowed \\
\hline Parent $T_{1 / 2}(\min )$ & 109.8 & 20.4 & 10.0 & 2.0 & 67.7 & 1.3 \\
\hline Daughter nucleus & ${ }^{18} \mathrm{O}$ & ${ }^{11} \mathrm{~B}$ & ${ }^{13} \mathrm{C}$ & ${ }^{15} \mathrm{~N}$ & ${ }^{68} \mathrm{Zn}$ & ${ }^{82} \mathrm{Kr}$ \\
\hline$E_{\max }(\mathrm{keV})$ & 633.5 & 960.2 & 1198.5 & 1732.0 & $821.8,1899.1$ & 2601,3378 \\
\hline Intensity & $97 \%$ & $99.8 \%$ & $99.8 \%$ & $99.9 \%$ & $1.2 \%, 87.9 \%$ & $13 \%, 82 \%$ \\
\hline$E_{\text {mean }}(\mathrm{keV})$ & 249.8 & 385.6 & 491.8 & 735.3 & $352.6,836.0$ & 1168,1535 \\
\hline Intensity & $97 \%$ & $99.8 \%$ & $99.8 \%$ & $99.9 \%$ & $1.2 \%, 87.9 \%$ & $13 \%, 82 \%$ \\
\hline $\begin{array}{l}\text { Intensity of } \\
\text { annihilation photons }\end{array}$ & $193.5 \%$ & $199.5 \%$ & $199.6 \%$ & $199.8 \%$ & $178.3 \%$ & $190.9 \%$ \\
\hline
\end{tabular}




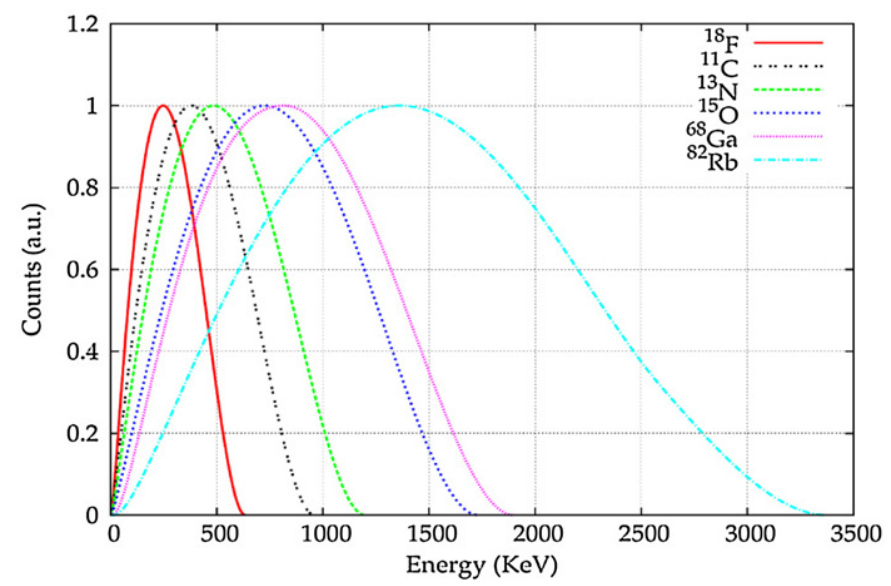

Figure 1. Simulated energy spectra for the radioisotopes considered in this work. The distributions are obtained with peneloPET following equation (1), and were normalized to the same value at the maximum.

\subsection{Positron annihilation}

When the positron reaches thermal velocities (a few eV), it either annihilates directly with an electron into two gamma rays, or first forms positronium $(\mathrm{Ps})$ in the singlet $\left({ }^{1} \mathrm{~S}_{0}\right.$, parapositronium, p-Ps) or triplet $\left({ }^{3} \mathrm{~S}_{1}\right.$, ortho-positronium, o-Ps) states (Berko and Hereford 1956). Para-positronium decays only into two photons, while ortho-positronium decays into three (Harpen 2004). The fraction of positrons forming positronium is known as the Ps yield. Using the technique of Positron Age-Momentum-Correlation (AMOC), Castellaz et al (2002) have measured Ps yields in a variety of liquids for an external $4 \mathrm{MeV}$ positron beam. The Ps yield in water was found to be $38 \%$ and in other liquids (organic solvents) it can be as high as $70 \%$ in spite of positron recoil effects at the liquid surface. It seems reasonable to expect the yield from lower energy sources embedded in tissue to be higher than the $38 \%$ found by Castellaz et al in water, thanks to the lack of surface and the lower instance of in-flight annihilation. In fact, Champion and Le Loirec $(2006,2007)$ estimated values of Ps yield of about $83 \%$ in water, independent on the isotope considered. Owing to the fact that positronium is a neutral particle and does not interact electromagnetically; the effect of Ps formation in the annihilation process will be a higher positron range. The subsequent positronium drift could be estimated from its residual momentum and effective half-life, as it has been described by Brawley et al (2010a, 2010b).

Once formed, p-Ps is observed to decay in liquids with its vacuum lifetime, 125 ps (Asai et al 1995). The observed lifetime of o-Ps in liquids is considerably shorter than its vacuum value due to pick-off effect, where a second electron with opposed spin reacts with the positron in the o-Ps atom resulting in two photon annihilation. The observed lifetime of o-Ps in water is $1800 \mathrm{ps}$, shorter than the vacuum lifetime of $140 \mathrm{~ns}$ (Westbrook et al 1989), but much longer than p-Ps lifetime, thus we may conclude that most annihilations in tissue result in two-gamma ray emissions despite the larger amount (3:1) of o-Ps initially formed (Harpen 2004).

While positron formation may be an important factor in positron range, in PeneloPET, as in the majority of other Monte Carlo codes, such as EGS4, Geant4, etc; positron annihilation is described as an in-flight process and formation of positronium is not considered. To the best of our knowledge, only in Champion and Le Loirec $(2006,2007)$ and Le Loirec and Champion 


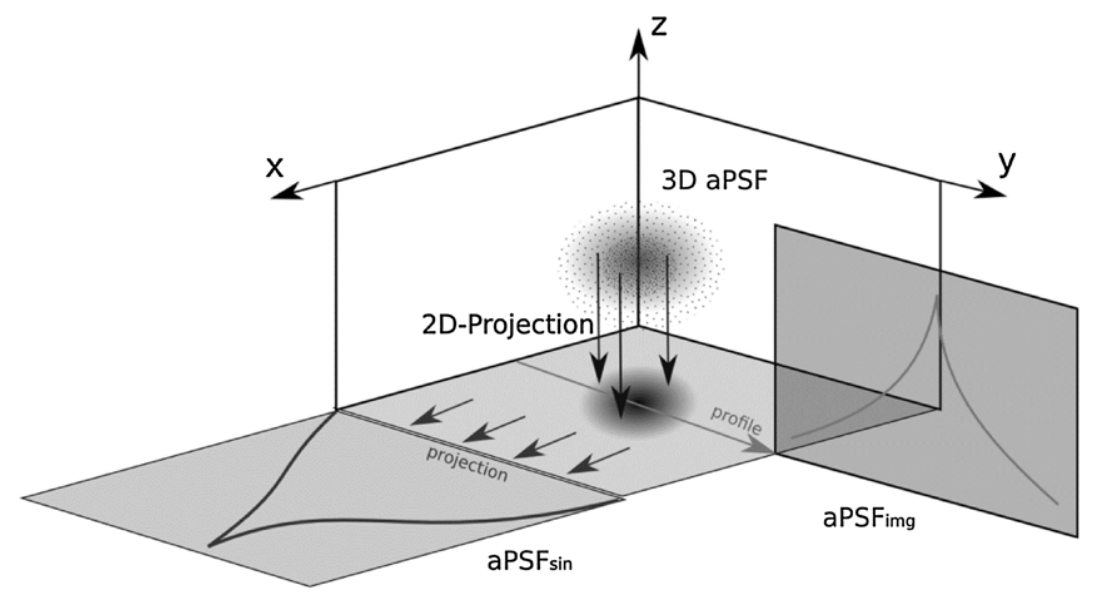

Figure 2. Schematic procedure to obtain $\mathrm{aPSF}_{\text {sin }}$ and $\mathrm{aPSF}_{\text {img }}$ from the original $3 \mathrm{D}$ aPSF.

(2007a, 2007b, 2007c), was Ps formation considered. When considering the effect of Ps in positron range, one must address Ps formation rates estimations inside tissues.

\section{Positron range modelling}

\subsection{Positron range distributions}

For a given positron emitting point source, the distribution of 3D Cartesian coordinates $(x, y, z)$ of the annihilation events provides the 3D annihilation point spread function (aPSF). A model for aPSF was proposed by Palmer and Brownell (1992) and by Palmer et al (2005). In those works, the aPSF for mono-energetic positrons (with energy $E_{0}<4 \mathrm{MeV}$ ) in isotropic media was represented by a three-dimensional Gaussian. Other authors compute 1D distribution from the aPSF. For instance, the projection onto one direction $\left(\mathrm{aPSF}_{\mathrm{sin}}\right)$, has been often employed. This distribution can be readily transformed into sinogram representation (Blanco 2006). $\mathrm{aPSF}_{\mathrm{sin}}$ distributions are employed for instance to report the results of the simulations of Levin and Hoffman (1999), Champion and Le Loirec (2007) and Blanco (2006). This 1D distribution is given by the expression:

$$
\operatorname{aPSF}_{\text {sin }}(x) \equiv \int_{-\infty}^{\infty} \mathrm{d} z \int_{-\infty}^{\infty} \operatorname{aPSF}(x, y, z) \mathrm{d} y
$$

A different 1D distribution can be formed by considering the profile, across the maximum, of the aPSF projected in a plane ( $\mathrm{aPSF}_{\mathrm{img}}$ ). This distribution is employed for instance in the experimental work of Derenzo (1979) and in simulations performed by Blanco (2006). It is given by:

$$
\operatorname{aPSF}_{\text {img }}(x) \equiv \int_{Y_{m}-\Delta y}^{Y_{m}+\Delta y} \mathrm{~d} y \int_{-\infty}^{\infty} \operatorname{aPSF}(x, y, z) \mathrm{d} z
$$

where $Y_{m}$ is the location of the maximum of the distribution projected in the plane and $\Delta y$ is the sampling interval on $y$. The schematic procedure to compute these 1D distributions starting from the original 3D aPSF is depicted in figure 2. $\mathrm{aPSF}_{\text {sin }}$ has been fit to a sum of two-exponential functions (Derenzo 1979):

$$
P(x)=C \mathrm{e}^{-k_{1} x}+(1-C) \mathrm{e}^{-k_{2} x} ; \quad x \geqslant 0, k_{1} \gg k_{2} ;
$$




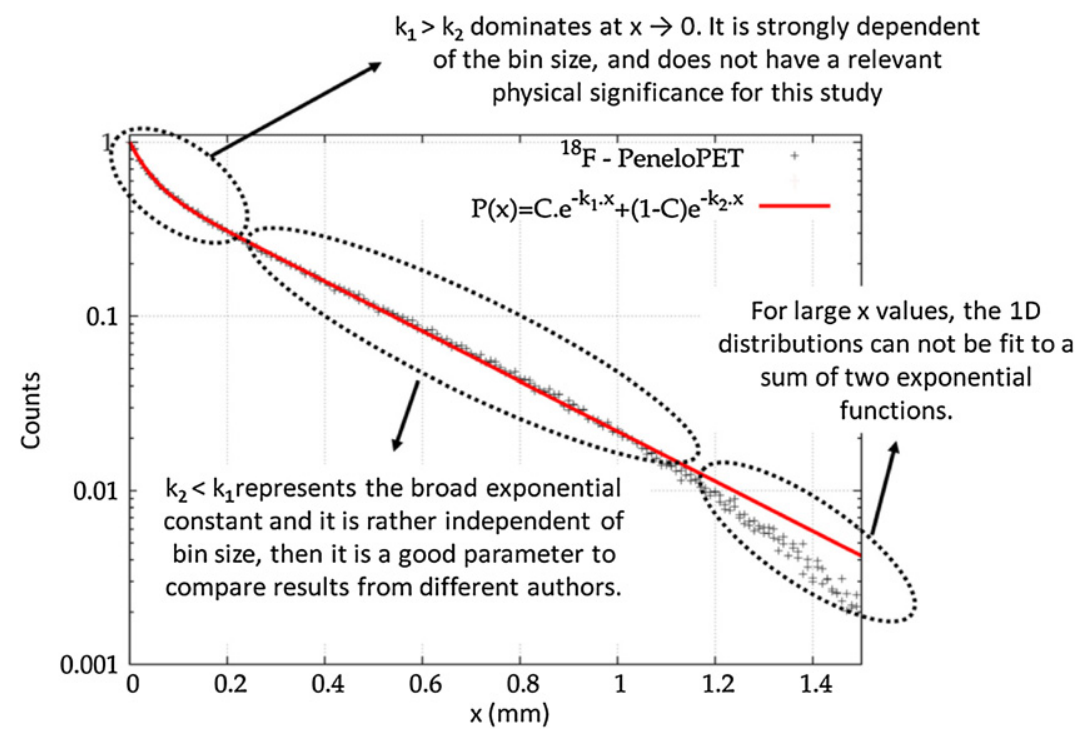

Figure 3. $\mathrm{aPSF}_{\text {sin }}$ obtained from a PeneloPET simulation of ${ }^{18} \mathrm{~F}$ in water and two-exponential fit proposed by Derenzo (1979).

Figure 3 sketches $\mathrm{aPSF}_{\text {sin }}$ for ${ }^{18} \mathrm{~F}$ point source in water. We can consider three different regions in the curve. For $x \rightarrow 0$, the distribution is determined by the $k_{1}$ coefficient. One has to take into account that in this limit the behaviour of the distribution depends strongly on the bin size chosen, thus $k_{1}$ should not be employed to compare to other results available in the literature. At intermediate values of $x$, the behaviour of the distribution is determined by $k_{2}$, that is a meaningful parameter to compare 1D distributions from different authors. Finally, for large $x$, comparable to the maximum positron range, the 1D distribution is no longer well represented by a sum of two-exponential functions, as it does not fall to zero for large distances, contrarily to actual positron range distributions.

In homogeneous media, $\operatorname{aPSF}(x, y, z)$ must be spherically symmetric for a point source. Thus it can be represented as $\operatorname{aPSF}(r)$. Therefore, 3D radial histograms can be also obtained for the positron annihilation distribution:

$$
\begin{aligned}
& g_{3 \mathrm{D}}(r) \equiv 4 \pi \int_{r}^{r+\mathrm{d} r} r^{\prime 2} \operatorname{aPSF}\left(r^{\prime}\right) \mathrm{d} r^{\prime} \\
& G_{3 \mathrm{D}}(r) \equiv \frac{\int_{0}^{r} g_{3 \mathrm{D}}\left(r^{\prime}\right) \mathrm{d} r^{\prime}}{\int_{0}^{\infty} g_{3 \mathrm{D}}\left(r^{\prime}\right) \mathrm{d} r^{\prime}}
\end{aligned}
$$

$g_{3 \mathrm{D}}(r)$ represents the number of annihilations at a given radial distance. The 3D cumulative distribution $G_{3 \mathrm{D}}(r)$ (equation $5 b$ ) can also be obtained from $\operatorname{aPSF}(r)$.

From the projection of aPSF $(r)$ in the $X Y$ plane, we can obtain 2D distribution $g_{2 \mathrm{D}}(r)$ and cumulative distribution $G_{2 \mathrm{D}}(r)$. These radial distributions can be obtained with the following expressions:

$$
\begin{aligned}
& g_{2 \mathrm{D}}(r) \equiv 2 \pi \int_{r}^{r+\mathrm{d} r} r^{\prime} \operatorname{aPSF}\left(r^{\prime}\right) \mathrm{d} r^{\prime} \\
& G_{2 \mathrm{D}}(r) \equiv \frac{\int_{0}^{r} g_{2 \mathrm{D}}\left(r^{\prime}\right) \mathrm{d} r^{\prime}}{\int_{0}^{\infty} g_{2 \mathrm{D}}\left(r^{\prime}\right) \mathrm{d} r^{\prime}}
\end{aligned}
$$


Table 2. Main relations and definitions of different positron range distributions found in the literature.

\begin{tabular}{ll}
\hline Relation & Mathematical expression \\
\hline $\mathrm{R} 1$ & $g_{3 \mathrm{D}}(r)=4 \pi \cdot r^{2} \cdot \mathrm{aPSF}_{3 \mathrm{D}}(r)$ \\
$\mathrm{R} 2$ & $\mathrm{aPSF}_{\mathrm{sin}}(r)=-\int_{0}^{r} \frac{1}{2 r^{\prime}} g_{3 \mathrm{D}}\left(r^{\prime}\right) \mathrm{d} r^{\prime}$ \\
$\mathrm{R} 3$ & $G_{3 \mathrm{D}}(r)=\int_{0}^{r} g_{3 \mathrm{D}}\left(r^{\prime}\right) \mathrm{d} r^{\prime} / \int_{0}^{\infty} g_{3 \mathrm{D}}\left(r^{\prime}\right) \mathrm{d} r^{\prime}$ \\
$\mathrm{R} 4$ & $\mathrm{aPSF}_{\mathrm{img}}(r)=\int_{\delta}^{\infty} \frac{1}{4 \pi \cdot r^{\prime}\left(r^{\prime 2}-\delta^{2}\right)^{1 / 2}} g_{3 \mathrm{D}}\left(r^{\prime}\right) \mathrm{d} r^{\prime}$ \\
$\mathrm{R} 5$ & $g_{2 \mathrm{D}}(r)=2 \pi \cdot r \cdot \mathrm{aPSF} \mathrm{img}(r)$ \\
$\mathrm{R} 6$ & $G_{2 \mathrm{D}}(r)=\int_{0}^{r} g_{2 \mathrm{D}}\left(r^{\prime}\right) \mathrm{d} r^{\prime} / \int_{0}^{\infty} g_{2 \mathrm{D}}\left(r^{\prime}\right) \mathrm{d} r^{\prime}$ \\
\hline
\end{tabular}

Champion and Le Loirec (2007) and Alessio and MacDonald (2008) showed 3D annihilation cumulative distributions $G_{3 \mathrm{D}}(r)$. Derenzo (1979) showed the 2D cumulative distributions $G_{2 \mathrm{D}}(r)$.

\subsection{Positron range modelling and simulation in PeneloPET}

PeneloPET was used to simulate the positron range of ${ }^{18} \mathrm{~F},{ }^{11} \mathrm{C},{ }^{13} \mathrm{~N},{ }^{15} \mathrm{O},{ }^{68} \mathrm{Ga}$ and ${ }^{82} \mathrm{Rb}$ in the following tissues: cortical bone, B-100 bone-equivalent plastic, skin, striated muscle, brain, water, adipose tissue and lung. Table 8 (section 7) shows the physical properties of these tissues, whose compositions were obtained from ICRU (1989) and ICRP (1975) databases. For each isotope/material combination investigated, more than five million positron histories were generated. $\operatorname{aPSF}_{\text {sin }}(x), \operatorname{aPSF}_{\mathrm{img}}(x), \operatorname{aPSF}(r), g_{3 \mathrm{D} / 2 \mathrm{D}}(r)$ and $G_{3 \mathrm{D} / 2 \mathrm{D}}(r)$ distribution profiles were histogrammed with a bin size of $10 \mu \mathrm{m}$.

\section{Comparison of positron range estimates}

\subsection{Comparison of positron range distributions using a genetic algorithm}

As many different ways of presenting positron range results are adopted in the literature, in order to facilitate the comparison of our simulations to previous results, we have developed a tool able of transforming a given positron range distribution into any other. We chose as canonical distribution the radial 3D distribution $g_{3 \mathrm{D}}(r)$. In this work we employed the following analytical expression for $g_{3 \mathrm{D}}(r)$ :

$$
g_{3 \mathrm{D}}(r) \approx C\left[(a \cdot r+1)\left[1-\frac{r}{r_{0}}\right]^{n}-\frac{\varepsilon}{r^{n}}\right]
$$

for $r<r_{0}$, and zero otherwise. $a, r_{0}, n$ and $\varepsilon(\varepsilon \ll 1)$ are fitting parameters which a genetic algorithm (see Fernández-Ramirez et al 2008) will modify to adjust $g_{3 \mathrm{D}}$ to data on whatever other quantity that can be derived from $g_{3 \mathrm{D}}$ (when needed, the relationships among different positron range distributions, table 2 and appendix, are employed). $\mathrm{C}$ is a constant employed to scale $g_{3 \mathrm{D}}$ to the data. In this work we present results scaled to a maximum value of one. By means of the genetic algorithm and the transformation among different profile expressions, results for range profile are employed to fit equation (7). Using $g_{3 \mathrm{D}}$ as canonical range distribution is convenient, but other distributions could be employed as well. For instance, the cumulative $G_{3 \mathrm{D}}$ is also a good indicator of positron range.

The analytical expression proposed in (7) has two parts. The first one represents the shape of the $g_{3 \mathrm{D}}(r)$ distribution for $r$ not close to 0 , while the second term, diverging at the origin, was added in order to give flexibility to fit to data when $r \rightarrow 0$. One has to recall that actually 


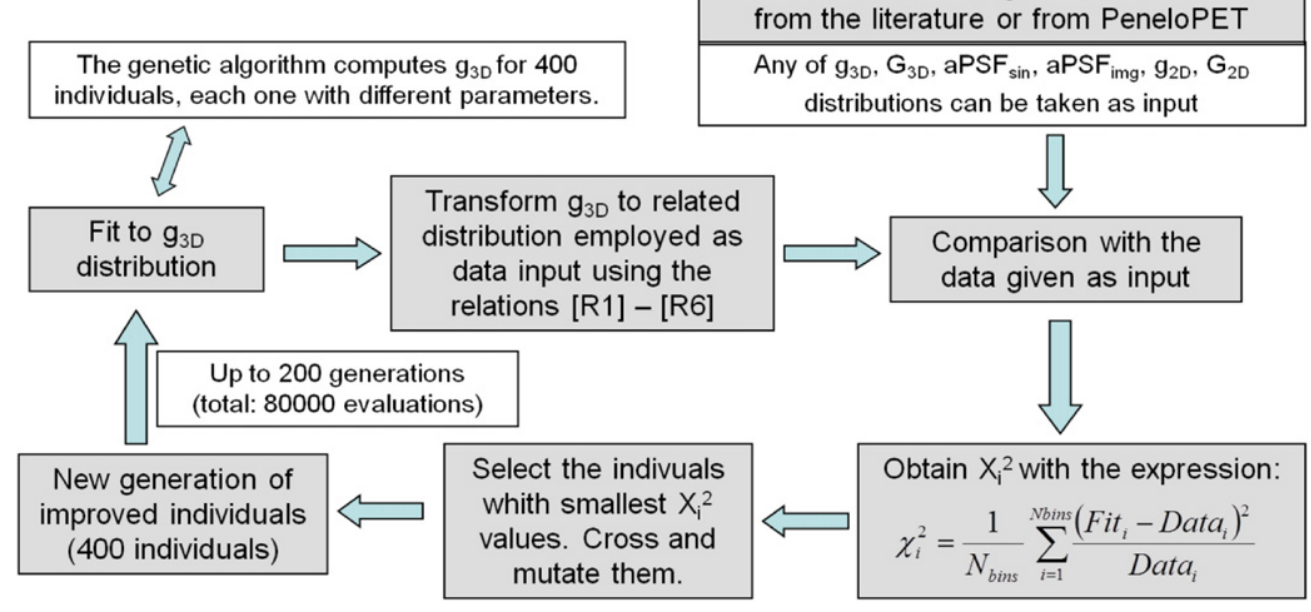

Figure 4. Procedure to fit positron range distributions.

the divergence near the origin displayed by equation (7) poses no special problem as long as the integral of equation (7) on any volume enclosing the origin, no matter how small, remains finite. This functional form makes it possible to fit projected profiles whose behaviour near the origin may depend on non-physical parameters, such as binning size. On the other hand, $r_{0}$ has a clear physical meaning: it represents the maximum positron range. Compared for instance to expression (3) previously mentioned and often employed in the literature, equation (7) shows the expected behaviour at large $r$. It has, however, one additional free parameter. The expression is just the result of several attempts to get a phenomenological fit to the observed range profiles imposing the condition for the profile to go to zero at a maximum range value.

As mentioned above, a genetic algorithm was used to fit $g_{3 \mathrm{D}}(r)$. The algorithm begins producing individuals with initial random values of the parameters, representing many possibilities for $g_{3 \mathrm{D}}(r)$. If needed, by means of relations (R1)-(R6) (see table 2), positron range distributions expressed in any of the usual ways found in the literature are obtained for each individual. The difference of the positron range distribution with the one taken as a reference is evaluated in terms of $\chi_{i}^{2}$ (see figure 4), that is employed as the fitness value for each individual. Individuals with small fitness are replaced by new ones. Evolution proceeds until a stable minimum $\chi_{i}{ }^{2}$ is obtained. This fitting procedure has proven to be very robust, and allows to obtain $g_{3 \mathrm{D}}(r)$ from positron range data expressed in any of the commonly found ways. Figure 4 shows a schematic diagram of the procedure employed. The genetic algorithm was running using 200 generations of 400 individuals each. This requires a few seconds on a modern personal computer. We found that the genetic algorithm produced adequate best fit parameters for all the profiles after single shot execution, without the need to prepare initial estimates of the parameters. This was not the case when a more standard optimization procedure, based on a steepest descent method, was tried instead.

\subsection{Comparison of mean and maximum positron range values}

We report (see tables 5 and 6, section 6.3) a comparison for PeneloPET to the available literature concerning mean and maximum positron ranges in water, $R_{\text {mean }}$ and $R_{\max }$, respectively. On the 
simulation side, we consider Champion and Le Loirec 2007, Partridge et al 2006 and Bailey

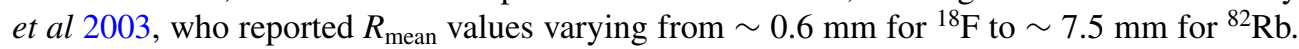
Concerning the maximum penetration of positrons in water, $R_{\max }$, we compare PeneloPET results with Champion and Le Loirec 2007. On the experimental side, Cho et al 1975 have measured range distributions for several nuclides. Additionally, we have also compared to positron range measurements taken from Derenzo (1979). We compare also with semiempirical expressions for the mean and maximum ranges. We consider the results of Katz and Penfold 1952, reviewed in Evans 1972. In his book, Evans estimates the mean positron range with the following semiempirical expression:

$$
R_{\text {mean }}(\mathrm{cm}) \approx \frac{0.108\left[E_{\beta}^{\max }(\mathrm{MeV})^{1.14}\right.}{\rho\left(\mathrm{g} \mathrm{cm}^{-3}\right)}
$$

The maximum positron range, also called extrapolated range, is very similar to the extrapolated range of electrons (see Evans 1972), and for energies between 0.01 and 2.5 $\mathrm{MeV}$, it can be estimated from the following semiempirical relationship:

$$
R_{\max }(\mathrm{cm}) \approx \frac{412\left[E_{\beta}^{\max }(\mathrm{MeV})^{. n}\right.}{\rho\left(\mathrm{mg} \mathrm{cm}^{-3}\right)} ; \quad 0.01 \leqslant \mathrm{E} \leqslant 2.5 \mathrm{MeV}
$$

With:

$$
\begin{aligned}
& n=1.265-0.0954 \ln E_{\beta}^{\max }(\mathrm{MeV}) \\
& R_{\max }(\mathrm{cm}) \approx \frac{530 E_{\beta}^{\max }(\mathrm{MeV})-106}{\rho\left(\mathrm{mg} \mathrm{cm}^{-3}\right)} ; \quad 2.5 \leqslant \mathrm{E} \leqslant 20 \mathrm{MeV} .
\end{aligned}
$$

\section{Annihilation distributions for PET isotopes using PeneloPET}

This section shows positron range distributions for the isotope-material combinations considered in this work.

\subsection{D Positron range distributions}

As we have already said, in order to obtain the $1 \mathrm{D}$ and $3 \mathrm{D}$ positron range distributions, more than five million positron histories were generated for each isotope/material. The bin size was $10 \mu \mathrm{m}$. We can estimate the expected error in the parameters of the simulated positron range distributions, from the variance of the Monte Carlo simulation. For instance, the expected error for the radial position at which $0.1 \%$ of the particles survive is less than $0.1 \%$ for short-range isotopes (as ${ }^{18} \mathrm{~F}$ or ${ }^{11} \mathrm{C}$ ) in water, less than $1 \%$ for large-range isotopes (as ${ }^{68} \mathrm{Ga}$ or ${ }^{82} \mathrm{Rb}$ ) in water, and less than $2 \%$ for long range isotopes in soft tissues, such as lung.

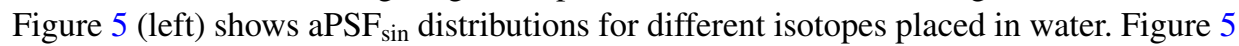
(right) shows the same distributions for ${ }^{18} \mathrm{~F}$ placed in different materials. Table 3 shows the values obtained for $k_{2}$ parameters for the $\mathrm{aPSF}_{\text {sin }}$ distributions and the isotopes studied in this work in the following materials: cortical bone, soft bone (B100 bone-equivalent plastic), water and lung.

As expected, $\mathrm{aPSF}_{\text {sin }}$ spans a larger spatial range for increasing energies of the positron and smaller spatial range for larger densities of the material in which the positron propagates and annihilates. 

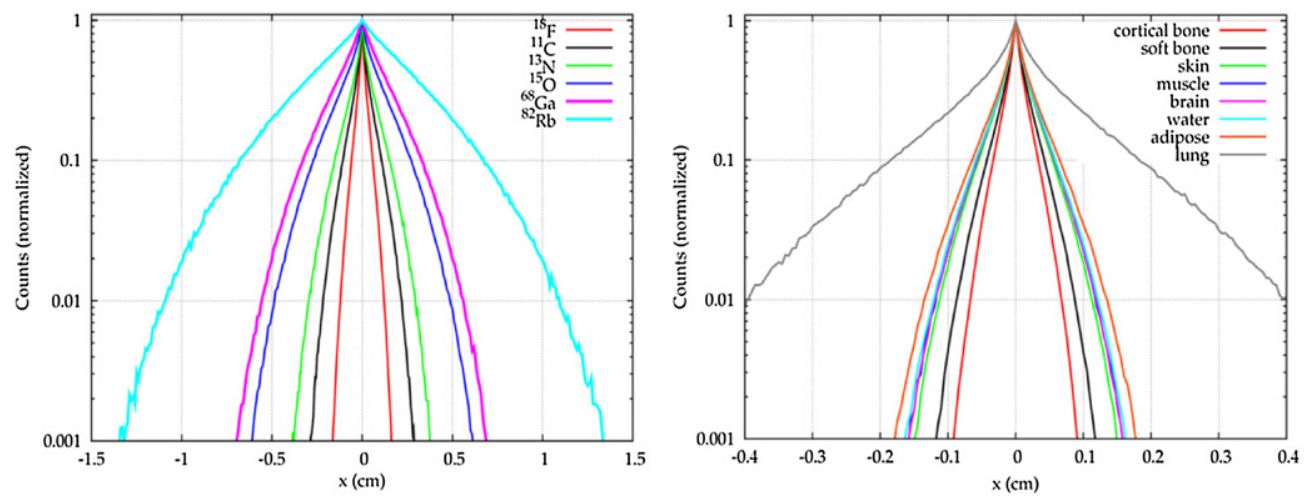

Figure 5. Left: aPSF sin distributions for ${ }^{18} \mathrm{~F},{ }^{11} \mathrm{C},{ }^{13} \mathrm{~N},{ }^{15} \mathrm{O},{ }^{68} \mathrm{Ga}$ and ${ }^{82} \mathrm{Rb}$ emitters in water. Right: $\mathrm{aPSF}_{\text {sin }}$ distributions for ${ }^{18} \mathrm{~F}$ emitter in different materials.
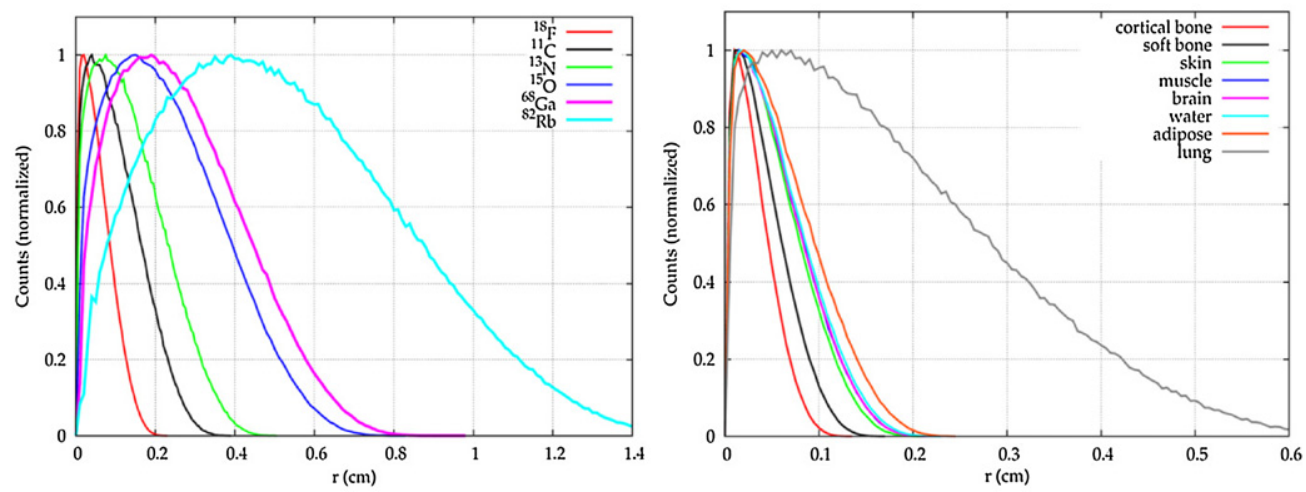

Figure 6. Left: $g_{3 \mathrm{D}}(r)$ distributions for ${ }^{18} \mathrm{~F},{ }^{11} \mathrm{C},{ }^{13} \mathrm{~N},{ }^{15} \mathrm{O},{ }^{68} \mathrm{Ga}$ and ${ }^{82} \mathrm{Rb}$ point sources in water. Right: $g_{3 \mathrm{D}}(r)$ distributions for a ${ }^{18} \mathrm{~F}$ point source in different materials.

Table 3. $k_{2}$ resulting from the fit to the positive $x$ region of the $\mathrm{aPSF}_{\text {sin }}$ distributions for each isotope in the following materials: cortical bone, soft bone, water and lung.

\begin{tabular}{lllll}
\hline Isotope & $\begin{array}{l}\text { Cortical bone } \\
k_{2}\left(\mathrm{~mm}^{-1}\right)\end{array}$ & $\begin{array}{l}\text { Soft bone } \\
k_{2}\left(\mathrm{~mm}^{-1}\right)\end{array}$ & $\begin{array}{l}\text { Water } \\
k_{2}\left(\mathrm{~mm}^{-1}\right)\end{array}$ & $\begin{array}{l}\text { Lung } \\
k_{2}\left(\mathrm{~mm}^{-1}\right)\end{array}$ \\
\hline${ }^{18} \mathrm{~F}$ & 6.06 & 4.69 & 3.27 & 0.98 \\
${ }^{11} \mathrm{C}$ & 3.29 & 2.54 & 1.79 & 0.53 \\
${ }^{13} \mathrm{~N}$ & 2.42 & 1.83 & 1.30 & 0.39 \\
${ }^{15} \mathrm{O}$ & 1.43 & 1.10 & 0.77 & 0.23 \\
${ }^{68} \mathrm{Ga}$ & 1.24 & 0.95 & 0.67 & 0.20 \\
${ }^{82} \mathrm{Rb}$ & 0.59 & 0.45 & 0.31 & 0.10 \\
\hline
\end{tabular}

\section{2. $3 D$ positron range distributions}

Radial histograms weighted by the total number of annihilations at a given radial distance $\left[g_{3 \mathrm{D}}(r)\right]$ and 3D cumulative distribution $G_{3 \mathrm{D}}(r)$ are studied in this section. Figure 6 shows 

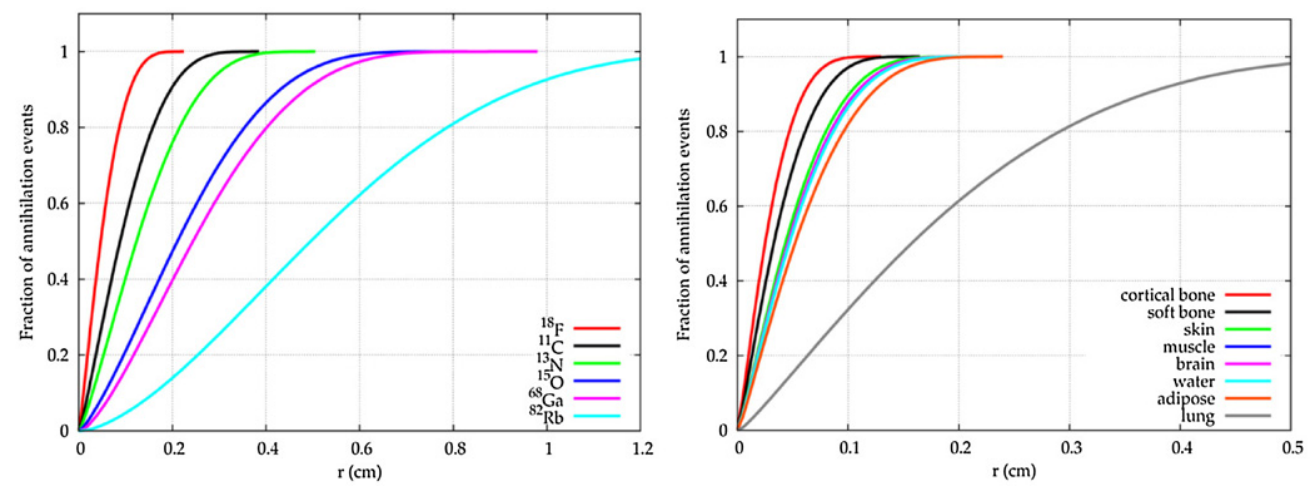

Figure 7. Left: $G_{3 \mathrm{D}}(r)$ distributions for ${ }^{18} \mathrm{~F},{ }^{11} \mathrm{C},{ }^{13} \mathrm{~N},{ }^{15} \mathrm{O},{ }^{68} \mathrm{Ga}$ and ${ }^{82} \mathrm{Rb}$ point sources in water. Right: $G_{3 \mathrm{D}}(r)$ distributions from a ${ }^{18} \mathrm{~F}$ point source in different materials.

Table 4. Comparison of the parameters resulting from the two-exponential fit for aPSF $\mathrm{sin}_{\text {in }}$ of PeneloPET (in bold) and the ones of Champion et al 2007 (1), Levin and Hoffman 1999 (2), Derenzo 1979 (3), Haber et al 1990 (4) and Blanco 2006 (5) for several isotopes in water. The parameter of the fit for the universal profile is also shown in the table.

\begin{tabular}{ll}
\hline & Water \\
Isotope & $k_{2}\left(\mathrm{~mm}^{-1}\right)$ \\
\hline${ }^{18} \mathrm{~F}$ & $\mathbf{3 . 2 7} / 2.79^{(1)} / 3.1^{(2)} / 3.94^{(4)} / 3.38^{(5)}$ \\
${ }^{11} \mathrm{C}$ & $\mathbf{1 . 7 9} / 1.49^{(1)} / 1.8^{(2)} / 2.19^{(3)} / 1.78^{(5)}$ \\
${ }^{13} \mathrm{~N}$ & $\mathbf{1 . 3 0} / 1.07^{(1)} / 1.4^{(2)} / 1.31^{(5)}$ \\
${ }^{15} \mathrm{O}$ & $\mathbf{0 . 7 7} / 0.60^{(1)} / 0.90^{(2)} / 0.77^{(5)}$ \\
${ }^{68} \mathrm{Ga}$ & $\mathbf{0 . 6 7} / 0.49^{(1)} / 0.87^{(3)} / 0.87^{(4)} / 0.65^{(5)}$ \\
${ }^{82} \mathrm{Rb}$ & $\mathbf{0 . 3 1} / 0.22^{(1)} / 0.33^{(3)} / 0.39^{(4)} / 0.30^{(5)}$ \\
Universal profile & $\mathbf{3 . 2 0}$ \\
\hline
\end{tabular}

$g_{3 \mathrm{D}}(r)$ for different isotopes placed in water (left) and $g_{3 \mathrm{D}}(r)$ for ${ }^{18} \mathrm{~F}$ placed in different materials (right). Equivalent plots are presented for $G_{3 \mathrm{D}}(r)$ in figure 7.

\section{Comparison of PeneloPET to previous results}

A comparison of PeneloPET results for positron range and other studies of positron range, either theoretical, simulated or experimental, presented in the literature is shown in this section. Wherever some observable for an author was not available, the genetic algorithm was employed to reconstruct $g_{3 \mathrm{D}}(r)$ and from it, other observables were derived.

\subsection{D distributions}

We will first consider $k_{2}$ from the two-exponentials fit to $\mathrm{aPSF}_{\text {sin }}$ given in table 4 . Figure 8 (left) shows the dependence of $k_{2}$ with the maximum $\beta^{+}$energy for the isotopes covered in this work. We find discrepancies with other results in the literature below $20 \%$, in the majority of cases. The dependence of $k_{2}$ on the density for a given isotope $\left({ }^{18} \mathrm{~F}\right.$ in this case) is shown in figure 8 (right). 

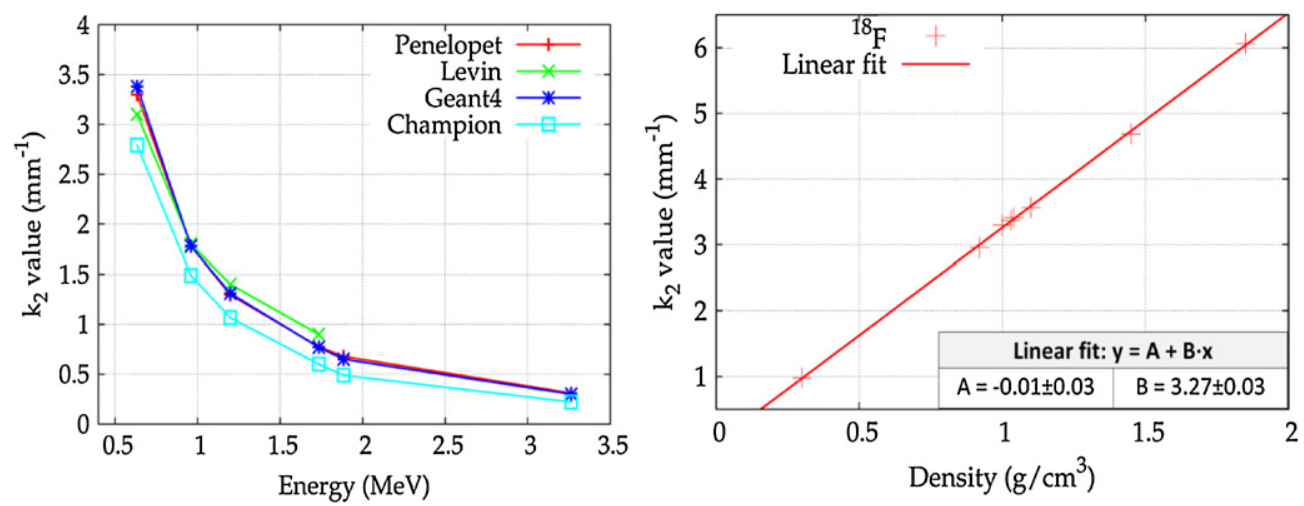

Figure 8. Left: dependence of $k_{2}$ with the maximum energy of the positron spectrum for each isotope. PeneloPET results are compared with results obtained with GEANT4 (Blanco 2006), Levin and Hoffman (1999), Derenzo (1979) and Champion and Le Loirec (2007). Right: dependence of $k_{2}$ with the density of the media, for ${ }^{18} \mathrm{~F}$.
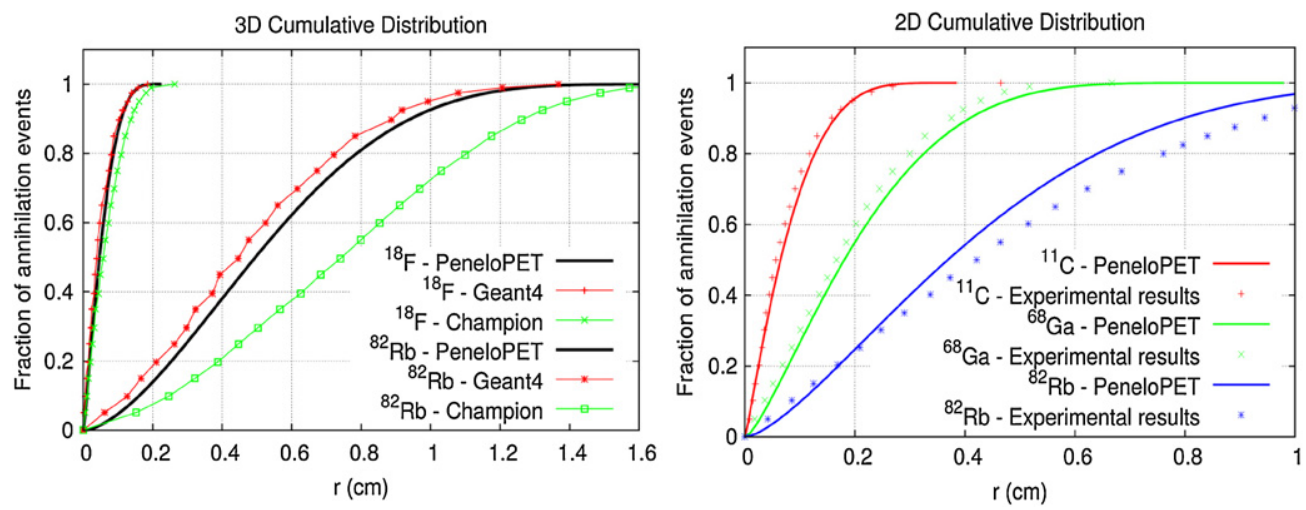

Figure 9. Left: 3D cumulative distributions obtained with PeneloPET, GEANT4 (Blanco 2006) and Champion and Le Loirec (2007) simulations, for ${ }^{18} \mathrm{~F}$ and ${ }^{82} \mathrm{Rb}$ isotopes in water. Right: $2 \mathrm{D}$ cumulative distributions for PeneloPET simulations compared to Derenzo (1979) experimental results, for ${ }^{11} \mathrm{C},{ }^{68} \mathrm{Ga}$ and ${ }^{82} \mathrm{Rb}$ isotopes in water.

Looking at figure 8, we note that PeneloPET estimations are in good agreement with the results of other authors, except for Champion's results, which under-estimate $k_{2}$ values compared to all other curves. This can be attributed to the consideration of positronium formation by Champion $e t$ al, while it is not included in all other calculations.

On the right side of the figure we can see that the dependence of $k_{2}$ on the density of the propagation media is almost linear.

\section{2. $2 D$ and $3 D$ cumulative distributions}

In this section we compare $G_{2 \mathrm{D}}(r)$ and $G_{3 \mathrm{D}}(r)$ distributions to the experimental results of Derenzo (1979) and with the simulations of Blanco (2006) and of Champion and Le Loirec (2007). Figure 9 shows these comparisons, for $G_{3 \mathrm{D}}(r)$ (left) and $G_{2 \mathrm{D}}(r)$ (right). As it was the case for $k_{2}$, PeneloPET results are relatively close to GEANT4 ones (they are within $12 \%$ of 
Table 5. Comparison of PeneloPET, GATE and semiempirical estimates from equation (8) for $R_{\text {mean }}$. Within parentheses are shown the deviations with respect to PeneloPET.

\begin{tabular}{|c|c|c|c|c|c|c|}
\hline \multirow[b]{2}{*}{ Isotope } & \multirow[b]{2}{*}{ Material } & \multicolumn{3}{|c|}{$R_{\text {mean }}(\mathrm{mm})$} & \multicolumn{2}{|c|}{$R_{\max }(\mathrm{mm})$} \\
\hline & & PeneloPET & GATE & Equation (8) & PeneloPET & Equation (9) \\
\hline \multirow[t]{3}{*}{${ }^{18} \mathrm{~F}$} & Cortical bone & 0.32 & $0.25(-21.9 \%)$ & $0.35(9.3 \%)$ & 1.28 & $1.24(-3.2 \%)$ \\
\hline & Water & 0.57 & $0.48(-15.8 \%)$ & $0.64(12.3 \%)$ & 2.16 & $2.27(4.8 \%)$ \\
\hline & Lung & 1.85 & $1.86(0.5 \%)$ & $2.14(15.7 \%)$ & 7.49 & $7.56(0.9 \%)$ \\
\hline \multirow[t]{3}{*}{${ }^{11} \mathrm{C}$} & Cortical bone & 0.55 & $0.48(-12.7 \%)$ & $0.56(1.8 \%)$ & 2.18 & $2.12(-2.8 \%)$ \\
\hline & Water & 1.02 & $0.92(-9.8 \%)$ & $1.03(1.0 \%)$ & 3.67 & $3.91(6.1 \%)$ \\
\hline & Lung & 3.35 & $3.61(7.8 \%)$ & $3.45(3.0 \%)$ & 12.4 & $13.0(4.6 \%)$ \\
\hline \multirow[t]{3}{*}{${ }^{13} \mathrm{~N}$} & Cortical bone & 0.75 & - & $0.72(-4.0 \%)$ & 2.82 & $2.79(-1.1 \%)$ \\
\hline & Water & 1.40 & $1.30(-7.1 \%)$ & $1.32(-5.7 \%)$ & 4.88 & $5.16(5.4 \%)$ \\
\hline & Lung & 4.61 & - & $4.43(-3.9 \%)$ & 16.1 & $17.2(6.4 \%)$ \\
\hline \multirow[t]{3}{*}{${ }^{15} \mathrm{O}$} & Cortical bone & 1.26 & $1.16(-7.9 \%)$ & $1.09(-13.5 \%)$ & 4.46 & $4.34(-2.8 \%)$ \\
\hline & Water & 2.34 & $2.21(-5.6 \%)$ & $2.01(-14.1 \%)$ & 7.92 & $8.02(1.2 \%)$ \\
\hline & Lung & 7.70 & $8.51(10.5 \%)$ & $6.73(-12.6 \%)$ & 24.7 & $26.7(7.5 \%)$ \\
\hline \multirow[t]{3}{*}{${ }^{68} \mathrm{Ga}$} & Cortical bone & 1.44 & - & $1.20(-16.7 \%)$ & 4.89 & $4.78(-2.3 \%)$ \\
\hline & Water & 2.69 & - & $2.24(-16.7 \%)$ & 9.06 & $8.84(-2.5 \%)$ \\
\hline & Lung & 8.86 & - & $7.41(-16.4 \%)$ & 27.1 & $29.5(8.1 \%)$ \\
\hline \multirow[t]{3}{*}{${ }^{82} \mathrm{Rb}$} & Cortical bone & 3.00 & - & $2.25(-25.0 \%)$ & 9.11 & $8.72(-4.5 \%)$ \\
\hline & Water & 5.33 & - & $4.29(-19.5 \%)$ & 16.5 & $16.1(-2.5 \%)$ \\
\hline & Lung & 17.6 & - & $13.90(-21.0 \%)$ & 52.0 & $53.8(3.3 \%)$ \\
\hline
\end{tabular}

each other) and to the experimental ones (only available in the literature for the 2D cumulative distribution, shown in the right part of figure 9), again with differences of the order of $10 \%$, while Champion's results depart from the ones obtained in this work by more than $25 \%$.

\subsection{Comparison of $R_{\text {mean }}$ and $R_{\text {max }}$ values}

Mean positron range values were computed with PeneloPET for different media and compared to GATE results (Lehnert et al 2011) and predictions of expression (8), reported by Evans (1972). This comparison is displayed in table 5. Table 6 shows mean and maximum positron ranges in water, $R_{\text {mean }}$ and $R_{\max }$ respectively.

\subsection{Comparison of positron range distributions using a genetic algorithm}

In the previous section we limited our comparison to observables actually quoted in the literature. Here we will use the genetic algorithm described before, to make a comparison of other observables related to positron range.

6.4.1. Fit of $g_{3 D}(r)$. First, we examine how well the genetic algorithm is able of reproducing $\mathrm{g}_{3 \mathrm{D}}(r)$ in a test case with simulated data from PeneloPET. Figure 10 shows the $g_{3 \mathrm{D}}(r)$ distribution fitted from the algorithm, using as input $g_{3 \mathrm{D}}$, $\mathrm{aPSF}_{\mathrm{Sin}}$ or $G_{3 \mathrm{D}}$ distributions. In all cases, the $g_{3 \mathrm{D}}(r)$ distribution obtained from the simulation is also plotted. These figures show that the analytical expression for $g_{3 \mathrm{D}}(r)$ proposed fits very well PeneloPET simulations.

Table 7 presents the resulting parameters of the fit of PeneloPET $g_{3 \mathrm{D}}(r)$ to equation (7), for the different isotopes considered in this work, annihilating in water. The expected error, resulting from the fit, in these parameters is less than $5 \%$ in all cases. It is important to keep in mind that the $r_{0}$ parameter in table 8 represents the maximum range of positrons. A comparison of $r_{0}$ to the maximum range derived from the PeneloPET simulations is included 
Table 6. PeneloPET results for $R_{\text {mean }}$ in water compared to Champion et al 2007 (1), Partridge et al 2006 (2), Bailey et al 2003(3) and for $R_{\max }$, to Champion et al 2007 (1). The table also shows semiempirical estimates from Evans (1972) (4) and measurements from Derenzo et al (1993) (5) and from Cho et al 1975 (6).

\begin{tabular}{|c|c|c|c|c|c|c|c|}
\hline \multicolumn{4}{|c|}{$R_{\text {mean }}$ in water $(\mathrm{mm})$} & \multicolumn{4}{|c|}{$R_{\max }$ in water $(\mathrm{mm})$} \\
\hline Isotopes & PeneloPET & Other simulations & Equation (8) & Experiment & PeneloPET & Other simulations & Equation (9) \\
\hline${ }^{18} \mathrm{~F}$ & 0.57 & $0.66^{(1)} / 0.6^{(2)} / 0.6^{(3)}$ & $0.64^{(4)}$ & $0.54^{(5)} / 0.9^{(6)}$ & 2.16 & $2.63^{(1)}$ & $2.27^{(4)}$ \\
\hline${ }^{11} \mathrm{C}$ & 1.02 & $1.13^{(1)} / 1.1^{(2)} / 1.1^{(3)}$ & $1.03^{(4)}$ & $0.92^{(5)} / 1.095^{(6)}$ & 3.67 & $4.54^{(1)}$ & $3.91^{(4)}$ \\
\hline${ }^{13} \mathrm{~N}$ & 1.40 & $1.73^{(1)} / 1.5^{(2)} / 1.5^{(3)}$ & $1.32^{(4)}$ & $1.39^{(6)}$ & 4.88 & $5.75^{(1)}$ & $5.16^{(4)}$ \\
\hline${ }^{15} \mathrm{O}$ & 2.34 & $2.96^{(1)} / 2.5^{(2)} / 2.5^{(3)}$ & $2.01^{(4)}$ & $1.785^{(6)}$ & 7.92 & $9.13^{(1)}$ & $8.02^{(4)}$ \\
\hline${ }^{68} \mathrm{Ga}$ & 2.69 & $3.56^{(1)} / 2.9^{(2)}$ & $2.24^{(4)}$ & $2.8^{(5)} / 1.975^{(6)}$ & 9.06 & $10.3^{(1)}$ & $8.84^{(4)}$ \\
\hline${ }^{82} \mathrm{Rb}$ & 5.33 & $7.49^{(1)} / 5.9^{(2)}$ & $4.29^{(4)}$ & $6.1^{(5)} / 2.9^{(6)}$ & 16.5 & $18.6^{(1)}$ & $16.1^{(4)}$ \\
\hline
\end{tabular}




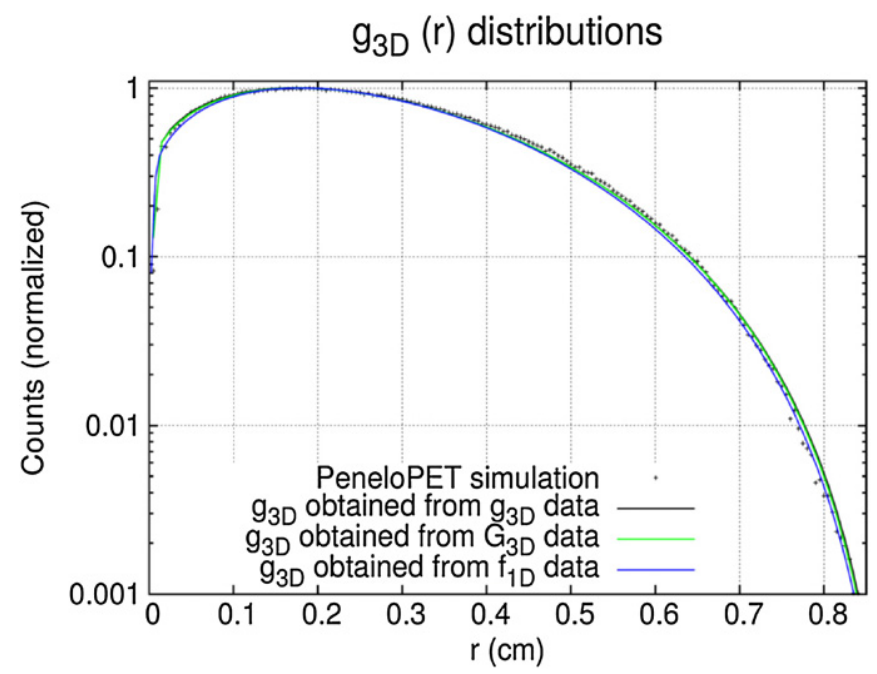

Figure 10. $g_{3 \mathrm{D}}(r)$ given by PeneloPET and the ones recovered with the genetic algorithm fitting equation (7) using as input $g_{3 \mathrm{D}}$, $\mathrm{APSF}_{\mathrm{sin}}$ or $G_{3 \mathrm{D}}$. For ${ }^{68} \mathrm{Ga}$ in water.

Table 7. Parameters of the fit to the $g_{3 \mathrm{D}}$ distribution of PeneloPET to equation (7).

\begin{tabular}{lllll}
\hline & \multicolumn{4}{l}{ Water. Number of positrons simulated: $5 \times 10^{6}$} \\
\cline { 2 - 5 } Isotope & $a\left(\mathrm{~mm}^{-1}\right)$ & $r_{0}(\mathrm{~mm}) / R_{\max }$ & $n$ & $r_{i}(\mathrm{~mm})$ \\
\hline${ }^{18} \mathrm{~F}$ & 2.60 & $2.39 / 2.16$ & 4.04 & 0.051 \\
${ }^{11} \mathrm{C}$ & 1.48 & $3.80 / 3.67$ & 3.16 & 0.21 \\
${ }^{13} \mathrm{~N}$ & 1.46 & $4.95 / 4.88$ & 3.04 & 0.29 \\
${ }^{15} \mathrm{O}$ & 1.15 & $7.90 / 7.92$ & 3.09 & 0.36 \\
${ }^{68} \mathrm{Ga}$ & 2.41 & $8.98 / 9.06$ & 3.27 & 0.20 \\
${ }^{82} \mathrm{Rb}$ & 7.13 & $16.6 / 16.5$ & 3.11 & 0.41 \\
Universal profile & $\mathbf{2 . 4 8}$ & $\mathbf{2 . 4 4 / 2 . 3 5}$ & $\mathbf{4 . 0 3}$ & $\mathbf{0 . 0 1 0 2}$ \\
\hline
\end{tabular}

in the table. On the other hand, $r_{i}$ is obtained using the following expression for $\varepsilon$ in expression (7) $\varepsilon \equiv 0.01 \times r_{i}^{n}$. In table 7 one can also see the fitting parameters for the universal profile computed in section 6 .

6.4.2. Comparison to range distributions from other authors. We compare PeneloPET positron range distributions with the ones presented by Alessio and MacDonald (2008), Derenzo (1979), Champion and Le Loirec (2007) and Levin and Hoffman (1999). In their paper, Alessio and MacDonald computed directly the $g_{3 \mathrm{D}}$ distributions for ${ }^{18} \mathrm{~F},{ }^{11} \mathrm{C},{ }^{13} \mathrm{~N}$ and ${ }^{82} \mathrm{Rb}$ isotopes annihilating in water. Derenzo calculated the $\mathrm{aPSF}_{\mathrm{img}}$ and $G_{2 \mathrm{D}}$ distributions for ${ }^{11} \mathrm{C},{ }^{68} \mathrm{Ga}$ and ${ }^{82} \mathrm{Rb}$ isotopes in water. Champion and Le Loirec computed the $g_{3 \mathrm{D}}, G_{3 \mathrm{D}}$ and $\mathrm{aPSF}_{\text {sin }}$ distributions for ${ }^{18} \mathrm{~F},{ }^{11} \mathrm{C},{ }^{13} \mathrm{~N},{ }^{15} \mathrm{O},{ }^{68} \mathrm{Ga}$ and ${ }^{82} \mathrm{Rb}$ isotopes, also in water. Finally, Levin and Hoffman obtained aPSF $\mathrm{sin}_{\text {in }}$ distributions for ${ }^{18} \mathrm{~F},{ }^{11} \mathrm{C},{ }^{13} \mathrm{~N}$ and ${ }^{15} \mathrm{O}$ isotopes.

Figure 11 shows $\operatorname{aPSF}_{\text {sin }}(x)$ for ${ }^{11} \mathrm{C},{ }^{13} \mathrm{~N},{ }^{68} \mathrm{Ga}$ and ${ }^{82} \mathrm{Rb}$ point sources in water. Figures 12 and 13 show the same plots for the $g_{3 \mathrm{D}}(r)$ and $G_{3 \mathrm{D}}(r)$ distributions. Alessio's (Alessio and MacDonald 2008) and Champion's (Champion and Le Loirec 2007) results were obtained 

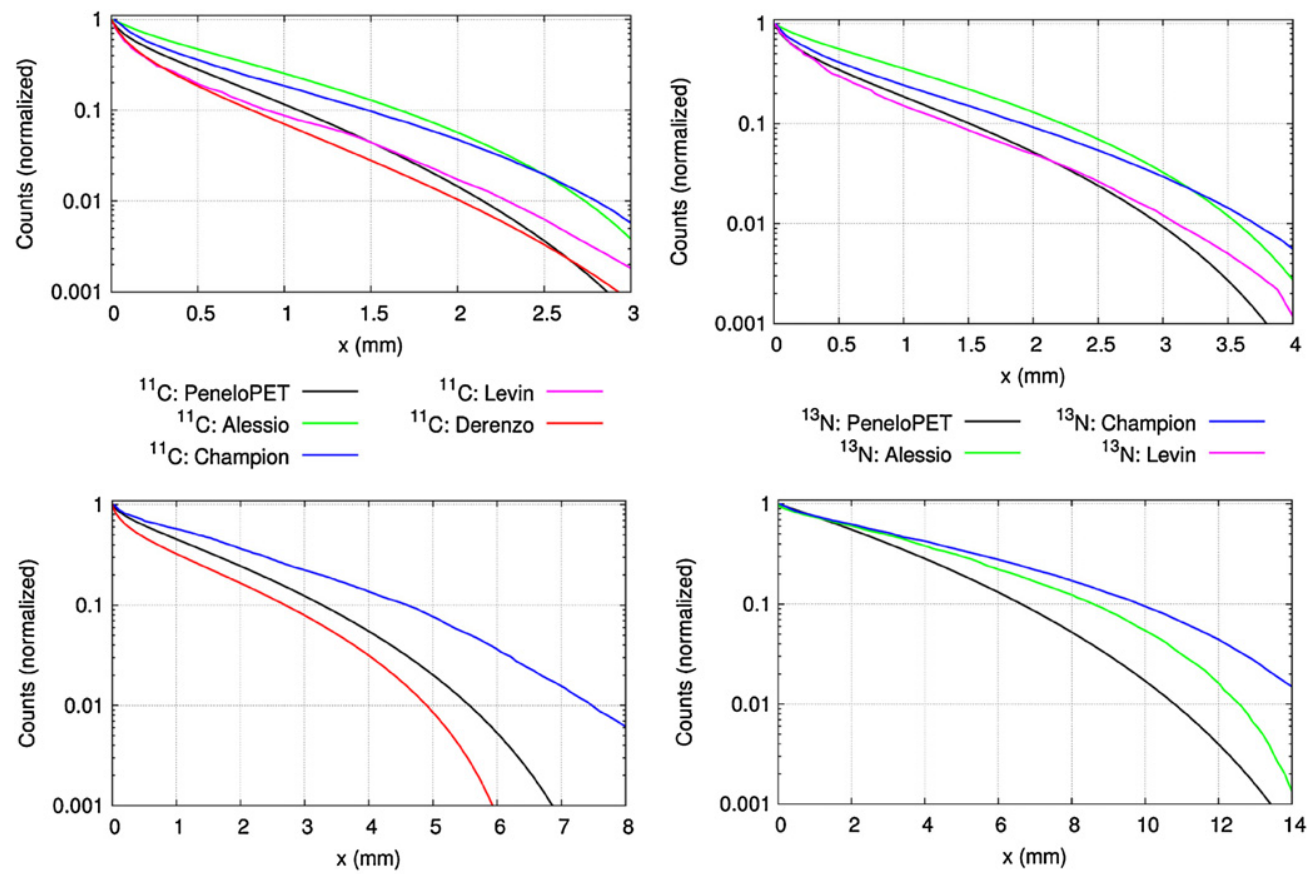

${ }^{68} \mathrm{Ga}$ PeneloPET — $\quad{ }^{68} \mathrm{Ga}$ : Champion -

${ }^{68} \mathrm{Ga}$ : Derenzo

${ }^{82} \mathrm{Rb}$ : PeneloPET

${ }^{82} \mathrm{Rb}$ : Alessio

Figure 11. Comparison of PeneloPET results with the ones of other authors (Alessio-green, Champion-blue, Levin — pink and Derenzo—red), obtained using the genetic algorithm, for the $\operatorname{aPSF}_{\sin }(x)$ distributions of ${ }^{11} \mathrm{C},{ }^{13} \mathrm{~N},{ }^{68} \mathrm{Ga}$ and ${ }^{82} \mathrm{Rb}$ isotopes annihilating in water.

Table 8. Comparison of PeneloPET mean and maximum positron ranges with the universally scaled prediction for the different isotopes and materials (differences in percentage are also shown).

\begin{tabular}{llllllc}
\hline & & \multicolumn{2}{c}{ Mean positron range $(\mathrm{mm})$} & & \multicolumn{2}{c}{ Maximum positron range (mm) } \\
Isotope & Material & $\begin{array}{l}\text { PeneloPET } \\
\text { simulation }\end{array}$ & $\begin{array}{l}\text { Universal } \\
\text { distribution }\end{array}$ & & $\begin{array}{l}\text { PeneloPET } \\
\text { simulation }\end{array}$ & $\begin{array}{l}\text { Universal } \\
\text { distribution }\end{array}$ \\
\hline${ }^{18} \mathrm{~F}$ & Adipose tissue & 0.62 & $0.62(-0.1 \%)$ & & 2.30 & $2.35(2.2 \%)$ \\
& B100 bone eq. plastic & 0.41 & $0.40(-2.9 \%)$ & & 1.55 & $1.49(-3.9 \%)$ \\
& Bone (cortical) & 0.32 & $0.31(-2.2 \%)$ & & 1.21 & $1.17(-3.3 \%)$ \\
& Brain & 0.55 & $0.55(0.0 \%)$ & & 2.10 & $2.08(-1.0 \%)$ \\
& Muscle & 0.55 & $0.55(0.2 \%)$ & & 2.06 & $2.08(1.0 \%)$ \\
& Lung & 1.85 & $1.91(3.6 \%)$ & & 7.41 & $7.20(-2.8 \%)$ \\
& Skin & 0.52 & $0.52(-0.5 \%)$ & & 2.00 & $1.96(-2.0 \%)$ \\
& Water & 0.57 & $0.57(1.2 \%)$ & & 2.16 & $2.16(0.0 \%)$ \\
${ }^{11} \mathrm{C}$ & Water & 1.02 & $1.03(1.2 \%)$ & & 3.67 & $3.56(-3.0 \%)$ \\
${ }^{13} \mathrm{~N}$ & Water & 1.40 & $1.41(1.3 \%)$ & & 4.88 & $4.86(-0.4 \%)$ \\
${ }^{15} \mathrm{O}$ & Water & 2.34 & $2.37(1.3 \%)$ & & 7.92 & $8.16(3.0 \%)$ \\
${ }^{68} \mathrm{Ga}$ & Water & 2.69 & $2.72(1.3 \%)$ & & 9.06 & $9.35(3.2 \%)$ \\
${ }^{82} \mathrm{Rb}$ & Water & 5.33 & $5.47(2.6 \%)$ & & 16.5 & $18.6(12.7 \%)$ \\
\hline
\end{tabular}

taking the $g_{3 \mathrm{D}}$ distribution from their published figures. On the other hand, Levin's (Levin and Hoffman 1999) results were obtained from the published $\mathrm{aPSF}_{\mathrm{sin}}$ distributions. Finally, Derenzo's (Derenzo 1979) results were obtained from the published $G_{2 \mathrm{D}}$ distributions. 

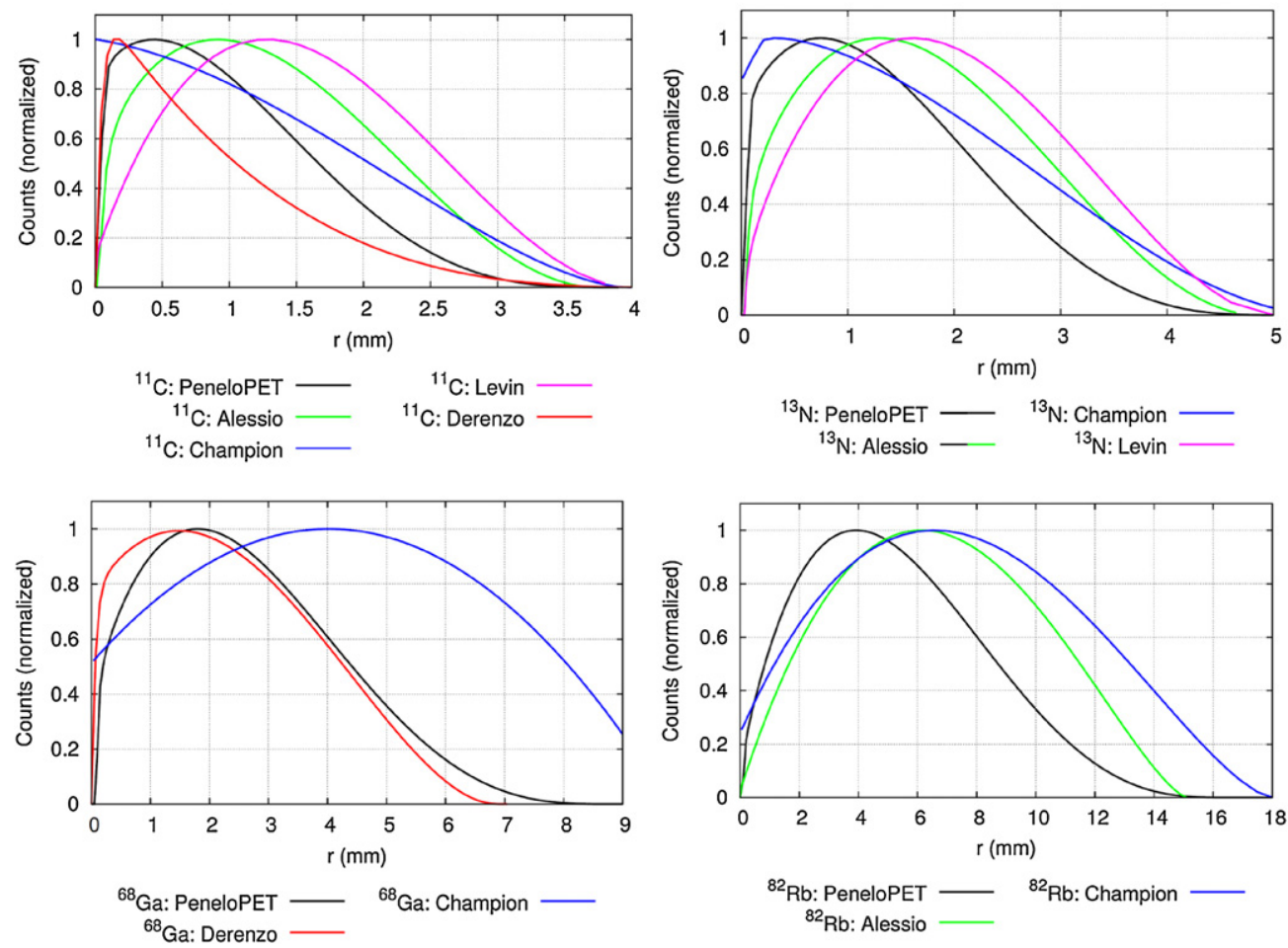

Figure 12. Comparison of PeneloPET results with the ones corresponding to other authors (Alessio—green, Champion—blue, Levin—pink and Derenzo—red), obtained using the genetic algorithm, for the $3 \mathrm{D}$ angular integrated radial distributions $\left[g_{3 \mathrm{D}}(r)\right]$ of ${ }^{11} \mathrm{C},{ }^{13} \mathrm{~N},{ }^{68} \mathrm{Ga}$ and ${ }^{82} \mathrm{Rb}$ isotopes annihilating in water.

Looking at figures 11-13, one can observe sizeable differences for $g_{3 \mathrm{D}}$ and $\mathrm{aPSF}_{\text {sin }}$ distributions from different authors. On the other hand, the 3D cumulative distributions $\left(G_{3 \mathrm{D}}\right)$ show smaller differences. Most distributions are well within $10 \%$ of each other, except, the ones from Champion and Le Loirec (2007). Champions' results are also the ones farthest away from Derenzo data.

\section{Scaling of positron range distributions}

Positron range profiles can be scaled to obtain universal profiles independent of the density of materials and energy of the emitter (Jødal et al 2012). To obtain material-independent profiles, a scaling by the density of material can be performed. The physical properties of these tissues were obtained from ICRU (1989) and ICRP (1975) databases.

A similar study of scaling with tissue density was recently presented by Jødal et al (2012). In this work we scale with the electronic density of the material instead, because we expect that positron range depends more linearly on relative electron density range are related by a more linear law than on tissue density.

To see whether a universal curve for different emitters in water is possible, and following a similar procedure than the one used by Jødal et al (2012), we scale also by the mean range of the positrons emitted by the isotope considered, previously calculated from PeneloPET simulations and quoted in table 8. 

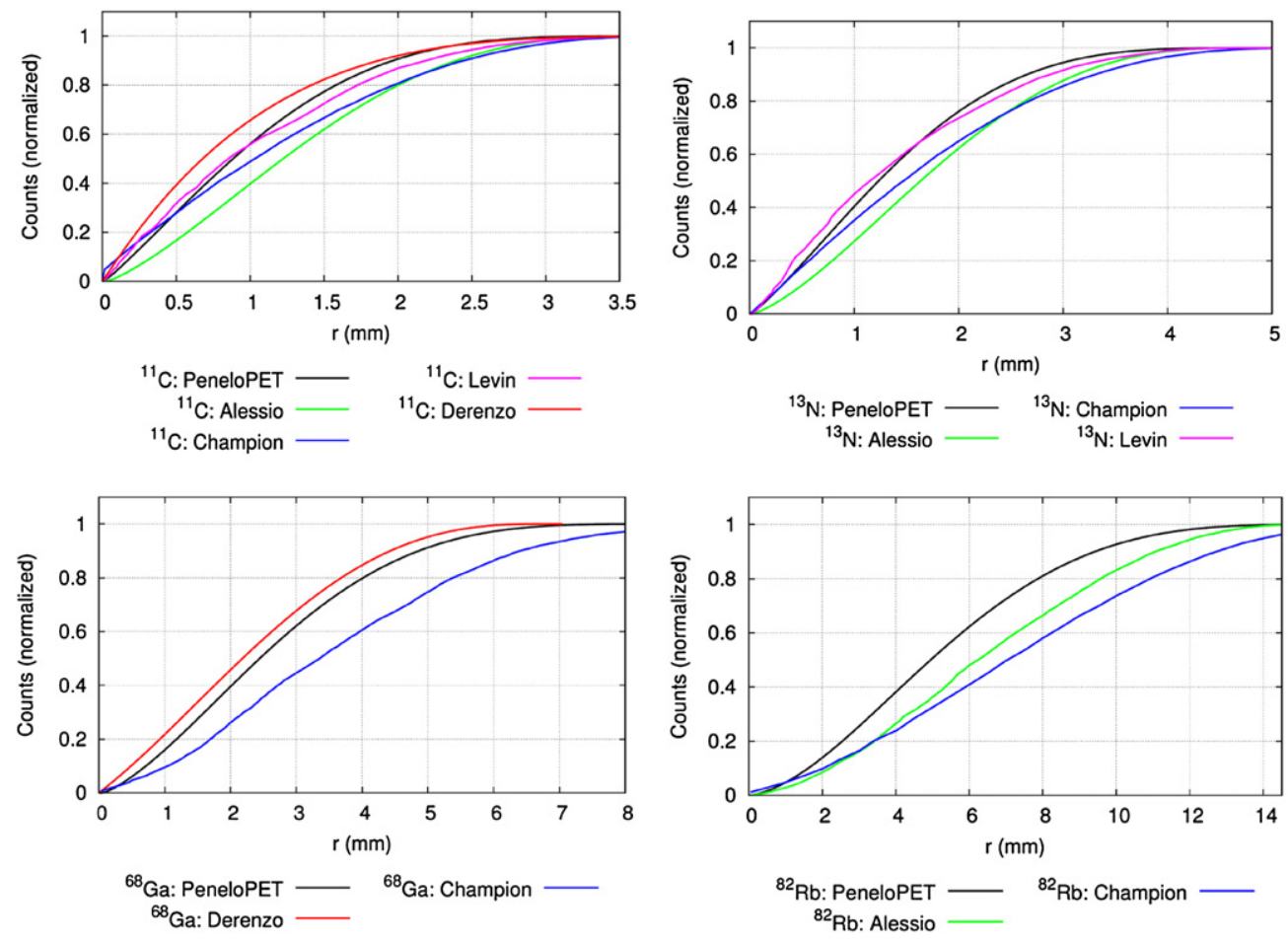

Figure 13. Comparison of PeneloPET results with the ones corresponding to other authors (Alessio-green, Champion-blue, Levin—pink and Derenzo-red), obtained using the genetic algorithm, for the $3 \mathrm{D}$ accumulated range distributions $\left[G_{3 \mathrm{D}}(r)\right]$ of ${ }^{11} \mathrm{C},{ }^{13} \mathrm{~N},{ }^{68} \mathrm{Ga}$ and ${ }^{82} \mathrm{Rb}$ isotopes annihilating in water.

Figure 14 (left) shows tissue-scaled aPSF $\mathrm{sin}_{3}, g_{3 \mathrm{D}}$ and $G_{3 \mathrm{D}}$ distributions for ${ }^{18} \mathrm{~F}$. The right side of the figure shows the range-scaled $\mathrm{aPSF}_{\mathrm{sin}}, g_{3 \mathrm{D}}$ and $G_{3 \mathrm{D}}$ distributions for different emitters placed in water and scaled by the mean range of the emitted positrons of each isotope. The universal curves, given by the average of the different tissue-scaled curves, are also shown in the figure. Table 8 shows mean and maximum ranges for different isotopes in water and for ${ }^{18} \mathrm{~F}$ in different materials. These mean ranges are also compared with the ones obtained from the universal curves. One can see that the mean and maximum positron ranges derived from the universal profiles, both for different emitters and different materials, are very similar to the ones obtained with PeneloPET, with discrepancies smaller than 5\% in almost all cases. Tissue-scaling in different biological materials, from cortical bone to lung tissue, as given in table 8 , is shown to be very good. Range profiles in different tissues can be described by a universal curve with less than a $4 \%$ error. Range-scaling is not as good as tissue-scaling for the emitters considered here. The $g_{3 \mathrm{D}}(r)$ distribution exhibits a larger sensitivity thus showing poorer range-scaling than the $1 \mathrm{D} \operatorname{aPSF}_{\text {sin }}(x)$ and the cumulative $G_{3 \mathrm{D}}(r)$ distributions.

\section{Discussion}

In this work we derived positron range distributions for different settings, and compared them to existing results in the literature. There is a significant diversity in the way different authors 

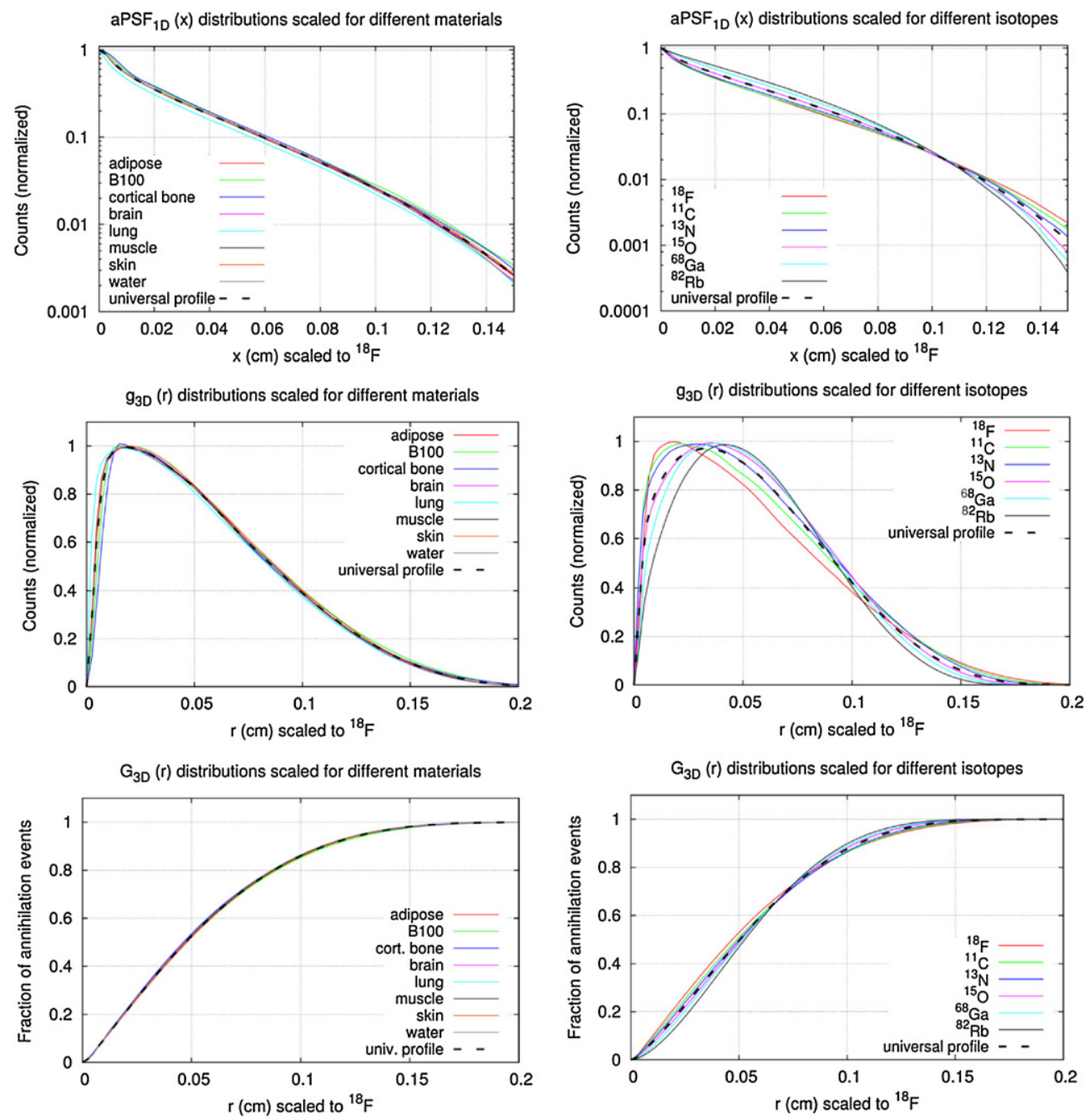

Figure 14. Left: scaled $\operatorname{aPSF}(x), g_{3 \mathrm{D}}(r)$ and $G_{3 \mathrm{D}}(r)$ distributions for ${ }^{18} \mathrm{~F}$ in different tissues. Right: comparison of different isotope distributions scaled by mean positron range.

present the results, for instance, using 1D projection of the positrons annihilation coordinates $\left(\mathrm{aPSF}_{\text {sin }}\right.$ and $\left.\mathrm{aPSF}_{\text {img }}\right)$, angular integrated radial distributions $\left(g_{3 \mathrm{D}}\right.$ and $\left.g_{2 \mathrm{D}}\right)$, or accumulated range distributions $\left(G_{3 \mathrm{D}}\right.$ and $\left.G_{2 \mathrm{D}}\right)$. It is known that differences of $10 \%$ in the mean and maximum positron range values lead to visible differences in range-corrected images (CalGonzález et al 2011). Although our estimations obtained with PeneloPET are in agreement with other works and with experimental measurements, there is a clear need for improved experimental results, in order to disentangle the role of positronium formation. Actually, calculations that take this phenomenon into account (Champion and Le Loirec 2007) clearly depart from most other results.

In our work we used a new procedure based on genetic algorithms to compare our results with others available in the literature. We fitted the $g_{3 \mathrm{D}}$ distribution to an expression with 3 free parameters (a fourth parameter is fixed by the profile normalization condition used in 
the positron range model). The parameter $r_{0}$ fits the maximum range, $n$ fits the slope at the end of the profile, and $a$ determines the slope at intermediate distances (equation 7). This fitting function outperforms the two-exponential sum proposed by Derenzo (1979) for the aPSF $_{\text {sin }}$, which fails for large $x$ values. Other authors (Champion and Le Loirec 2007, Lehnert et al 2011) proposed a Gaussian fit for $g_{3 \mathrm{D}}$, with only three parameters. However, PeneloPET results show that these functions depart from the Gaussian shape for large $r$ values. More recently, Jødal et al (2012) proposed a non-Gaussian fit that seems to perform similarly to ours. However, their fit does not include any parameter explicitly related to maximum positron range. As expected, $g_{3 \mathrm{D}}$ and $\mathrm{aPSF}_{\text {sin }}$ distributions tend to be more different for several authors and approaches, while 3D cumulative distributions $\left(G_{3 \mathrm{D}}\right)$ are more stable. $G_{3 \mathrm{D}}$ represents the fraction of positrons annihilated within a sphere or radius $r$ and it seems to be more robust to compare results available in the literature.

We also compared the mean and maximum positron ranges in water obtained with PeneloPET with those previously available in the literature (tables 5 and 6). Regarding the mean range $R_{\text {mean }}$, a relatively good agreement is observed between our results and other simulated or semiempirical estimates, with discrepancies ranging from $7 \%$ for ${ }^{18} \mathrm{~F}$ to $18 \%$ for ${ }^{82} \mathrm{Rb}$. However, the differences with the results of Champion and Le Loirec (2007) are larger, up to $40 \%$. Further, they are larger for larger positron range isotropes. These discrepancies are likely due to the fact that Positronium formation is included in Champion's results, in contrast with our calculations and most other results that do not consider it. Regarding to experimental data, PeneloPET predictions for ${ }^{18} \mathrm{~F}$ are in disagreement with the $R_{\text {mean }}$ values measured by Cho et al (1975) by more than $50 \%$ for ${ }^{18} \mathrm{~F}$, but they are in relatively good agreement with the data provided by Derenzo (1979). The discrepancies observed between our results and some experimental data are likely due to the limited accuracy achievable in the experimental procedures, since the detector resolution was comparable to the positron range for the lowest range isotopes, such as ${ }^{18} \mathrm{~F}$. Regarding the maximum range, $R_{\max }$, PeneloPET predictions are consistent with those obtained from Evans' empirical relationships (Evans 1972), while the discrepancies with Champion and Le Loirec (2007) are substantial. Table 5 shows the comparison of $R_{\text {mean }}$ values obtained for different media with the results obtained with GATE simulations and the theoretical predictions given by the expression in equation (8). Although PeneloPET and GATE results are in good agreement, with discrepancies smaller than $10 \%$ in almost all cases, larger relative differences can be observed for the higher range isotopes: ${ }^{15} \mathrm{O}$, ${ }^{68} \mathrm{Ga}$, and ${ }^{82} \mathrm{Rb}$.

As it was anticipated in some recent works (Jødal et al 2012), positron range distributions predicted by PeneloPET after scaling by material densities yield very similar results for different materials, with differences in the mean and maximum range values smaller than $4 \%$ in most cases. Scaling by the mean range of the positron emitter is less accurate. For the allowed decays considered here, scaling for different isotopes produces differences of about $5 \%$, except for ${ }^{82} \mathrm{Rb}$, which shows discrepancies of about $12 \%$ in the maximum range (see table 8 ). These two scaling rules would enable a reasonably accurate estimation of positron range distributions for any isotope/material combination, and may play a key role for fast algorithms aimed to correct images for positron range during reconstruction (Cal-González et al 2011). The parametric expression for the universal scaled curves proposed in equation (7) would facilitate these implementations.

Positronium (Ps) formation deserves a deeper discussion. As mentioned above, the results of the work that considered Ps formation (Champion and Le Loirec 2007) depart from all the other ones, which are otherwise in relatively good agreement with each other. This is somehow understandable since Ps formation is expected to have a clear impact on the positron range, especially for formation probabilities larger than $80 \%$, such as the ones used in Champion and 
Le Loirec (2007). However, these results, that include Ps effects, do not match the experimental results of Derenzo (1979) while it is unclear for the experimental values reported by Cho et al (1975), which are in turn very different from the Derenzo's ones for the larger range isotopes. It is noteworthy that the improvement of the physical model by considering Ps formation seems to worsen the agreement with experiments, especially for larger range isotopes. This may be due to different Ps formation probabilities for different materials. While Champion and Le Loirec (2007) values were extracted from simulations in water, Derenzo experiment was performed in low-density polyurethane foam. Positronium formation probabilities might be very different in liquid water than in the foam. In consequence, while the inclusion of positronium effects in a simulation may be relatively straightforward, we must realize that accuracy can only be achieved by knowing the formation probabilities for most body tissues, or at least for bone, water and lung. Furthermore, Ps formation (and the ratio of o-Ps and p-Ps) would not scale with electron density or tissue density as positron range does, because it also depends on the momentum distribution of the electrons in the medium. In this way, the scaling of positron range for different tissues should not be considered accurate until the issue of Ps formation is solved. In order to complete the model and to achieve a thorough description of the phenomena involved, accurate experimental measurements of positron range in relevant biological tissues and Ps formation (and Ps lifetimes) are warranted.

\section{Summary and conclusions}

In this work we used PeneloPET to generate Monte Carlo simulations of positron range for different radioisotopes in different media. $\mathrm{aPSF}_{\mathrm{sin}}, \mathrm{aPSF}_{\mathrm{img}}, g_{3 \mathrm{D}}, g_{2 \mathrm{D}}, G_{3 \mathrm{D}}$ and $G_{2 \mathrm{D}}$ distributions for each combination isotope/material were determined and PeneloPET predictions were compared with values from previous literature. To this end, we also propose the use of a new framework based on a genetic algorithm that fits the radial, angle integrated, $g_{3 \mathrm{D}}(r)$ distribution of the annihilation points, to any distribution chosen as reference.

Our results were consistent with previous literature, except for the case of Champion and Le Loirec (2007), which included positronium formation in their model. Insufficient experimental data are available to properly assess the importance of positronium formation. Therefore, more accurate experimental results for positron range, especially on newer isotopes and in biological tissues are needed in order to correct for range effects, especially in preclinical image reconstruction.

Finally, the scaling method to estimate the positron range distributions for any isotope/material combination was confirmed according to PeneloPET simulations. Scaling by the density of media has been shown to work well, and scaling for different isotopes allows estimating positron range distributions with acceptable accuracy in most cases.

\section{Acknowledgments}

We kindly acknowledge support from Comunidad de Madrid (ARTEMIS S2009/DPI-1802), Spanish Ministry of Science and Innovation (grants FPA2010-17142 and ENTEPRASE, PSE300000-2009-5), by European Regional Funds, by CDTI under the CENIT Programme (AMIT Project) and by CPAN, CSPD-2007-00042@Ingenio2010. Part of the calculations of this work were performed in the 'Clúster de Cálculo de Alta Capacidad para Técnicas Físicas' funded in part by UCM and in part by UE under FEDER programme. This is a contribution to the Campus of International Excellence of Moncloa. 


\section{Appendix. Relations among different positron range distributions}

$g_{3 \mathrm{D}}(r)$ was expressed in section 2.5 as:

$$
g_{3 \mathrm{D}}(r)=4 \pi \int_{r}^{r+\mathrm{d} r} r^{\prime 2} \operatorname{aPSF}\left(r^{\prime}\right) \mathrm{d} r^{\prime}
$$

Assuming isotropy, we can consider the range distribution without integration in angles $\left(f_{3 \mathrm{D}}(r)\right)$, and obtain the following relationship:

$$
g_{3 \mathrm{D}}(r)=4 \pi r^{2} \mathrm{aPSF}_{3 \mathrm{D}}(r)
$$

Following the relations proposed in Poularikas (2000), the relationship between the 3D and $1 \mathrm{D}$ distribution becomes:

$$
\operatorname{aPSF}_{3 \mathrm{D}}(r)=-\frac{1}{2 \pi \cdot r} \mathrm{~d}\left[\operatorname{aPSF}_{\sin }(r)\right] / \mathrm{d} r
$$

Using (R1) and (R2) we can relate $\operatorname{aPSF}_{\text {sin }}(r)$ and $g_{3 \mathrm{D}}(r)$ :

$$
\operatorname{aPSF}_{\text {sin }}(r)=-\int_{0}^{r} \frac{1}{2 r^{\prime}} g_{3 \mathrm{D}}\left(r^{\prime}\right) \mathrm{d} r^{\prime}
$$

The cumulative 3D distribution $G_{3 \mathrm{D}}(r)$ was obtained as a function of $g_{3 \mathrm{D}}(r)$ :

$$
G_{3 \mathrm{D}}(r)=\frac{\int_{0}^{r} g_{3 \mathrm{D}}\left(r^{\prime}\right) \mathrm{d} r^{\prime}}{\int_{0}^{\infty} g_{3 \mathrm{D}}\left(r^{\prime}\right) \mathrm{d} r^{\prime}}
$$

For the $\mathrm{APSF}_{\mathrm{img}}$ and 2D distributions the Abel transform was used (see Poularikas 2000_ chapter 8):

$$
\operatorname{aPSF}_{\mathrm{img}}(r)=\int_{\delta}^{\infty} \frac{1}{4 \pi \cdot r^{\prime}\left(r^{\prime 2}-\delta^{2}\right)^{1 / 2}} g_{3 \mathrm{D}}\left(r^{\prime}\right) \mathrm{d} r^{\prime}
$$

$g_{2 \mathrm{D}}(r)$ can be obtained from $\operatorname{aPSF}_{\mathrm{img}}(r)$ :

$$
g_{2 \mathrm{D}}(r) \equiv 2 \pi \cdot r \cdot \operatorname{aPSF}_{\mathrm{img}}(r)
$$

Finally, the cumulative 2D distribution $G_{2 \mathrm{D}}(r)$ was already expressed in terms of $g_{2 \mathrm{D}}(r)$ in section 2.5:

$$
G_{2 \mathrm{D}}(r)=\frac{\int_{0}^{r} g_{2 \mathrm{D}}\left(r^{\prime}\right) \mathrm{d} r^{\prime}}{\int_{0}^{\infty} g_{2 \mathrm{D}}\left(r^{\prime}\right) \mathrm{d} r^{\prime}}
$$

Using (R1)-(R6), $g_{3 \mathrm{D}}(r)$ can be related to any other positron range distribution.

\section{References}

Agostinelli S 2003 GEANT4-a simulation toolkit Nucl. Instrum. Methods Phys. Res. A 506 250-303

Alessio A and MacDonald L 2008 Spatially variant positron range modeling derived from CT for PET image reconstruction IEEE Nucl. Sci. Symp. Conf. Rec. pp 3637-40

Allison J et al 2006 Geant4 developments and applications IEEE Trans. Nucl. Sci. 53 270-8

Andreo P and Brahme A 1984 Restricted energy-loss straggling and multiple scattering of electrons in mixed Monte Carlo procedures Radiat. Res. 100 16-29

Asai S, Orito S and Shinohara N 1995 New measurement of the orthopositronium decay rate Phys. Lett. B 357 475-80

Bai B, Laforest R, Smith A M and Leahy R M 2005 Evaluation of MAP image reconstruction with positron range modeling for 3D PET IEEE Nucl. Sci. Symp. Conf. Rec. pp 2686-9

Bai B, Ruangma A, Laforest R, Tai Y C and Leahy R M 2003 Positron range modeling for statistical PET image reconstruction IEEE Nucl. Sci. Symp. Conf. Rec. pp 2501-5

Bailey D L, Karp J S and Surti S 2003 Physics and instrumentation in PET Positron Emission Tomography: Basic Science and Clinical Practice ed P E Valk et al (London: Springer) pp 41-67

Baró J, Sempau J, Fernández-Varea J M and Salvat F 1995 PENELOPE: an algorithm for Monte Carlo simulation of the penetration and energy loss of electrons and positrons in matter Nucl. Instrum. Methods Phys. Res. B $10031-46$ 
Berko S and Hereford FL 1956 Experimental studies of positron interactions in solids and liquids Rev. Mod. Phys. 28 299-307

Bielajew A F and Salvat F 2001 Improved electron transport mechanics in the PENELOPE Monte-Carlo model Nucl. Instrum. Methods B 173 332-43

Blanco A 2006 Positron range effects on the spatial resolution of RPC-PET IEEE Nucl. Sci. Symp. Conf. Rec. pp 2570-3

Brawley S J, Armitage S, Beale J, Leslie D E, Williams A I and Laricchia G 2010a Electron-like scattering of positronium Science 330789

Brawley S J, Williams A I, Shipman M and Laricchia G 2010b Resonant scattering of positronium in collision with $\mathrm{CO}_{2}$ Phys. Rev. Lett. 105263401

Breeman W A P and Verbruggen A M 2007 The ${ }^{68} \mathrm{Ge} /{ }^{68} \mathrm{Ga}$ generator has high potential, but when can we use ${ }^{68} \mathrm{Ga}$-labelled tracers in clinical routine? Eur. J. Nucl. Med. Mol. Imaging 34 978-81

Cal-González J, Herraiz J L, España S, Vicente E, Herranz E, Desco M, Vaquero J J and Udias J M 2011 Study of CT-based positron range correction in high resolution 3D PET imaging Nucl. Instrum. Methods Phys. Res. A 172-5

Castellaz P, Siegle A and Stoll H 2002 Positron age-momentum correlation (AMOC) measurements on organic liquids J. Nucl. Radiochem. Sci. 3 R1-7 (available at http://pubman.mpdl.mpg.de/pubman/faces/ viewItemFullPage.jsp?itemId=escidoc:1573943:1)

Champion C and Le Loirec C 2006 Positron follow-up in liquid water: I. A new Monte Carlo track-structure code Phys. Med. Biol. 51 1707-23

Champion C and Le Loirec C 2007 Positron follow-up in liquid water: II. Spatial and energetic study for the most important radioisotopes used in PET Phys. Med. Biol. 52 6605-25

Cho Z H, Chan J K, Ericksson L, Singh M, Graham S, Macdonald N S and Yano Y 1975 Positron ranges obtained from biomedically important positron-emitting radionuclides J. Nucl. Med. 16 1174-6 PMID: 1194970

Derenzo S E 1979 Precision Measurement of Annihilation Point Spread Distributions for Medically Important Positron Emitters, Positron Annihilation ed R R Hasiguti and K Fujiwara (Sendai: The Japan Institute of Metals) pp 819-23 (available at www.osti.gov/bridge/product.biblio.jsp?osti_id=6222101)

Derenzo S E 1986 Mathematical removal of positron range blurring in high resolution tomography IEEE Trans. Nucl. Sci. 33 565-9

Derenzo S E, Moses W W, Huesman R H and Budinger F B 1993 PET 93 'ICS 1030' (Akita, Japan, 29-31 May)

España S, Herraiz J L, Vicente E, Vaquero J J, Desco M and Udias J M 2009 PeneloPET, a Monte Carlo PET simulation tool based on PENELOPE: features and validation Phys. Med. Biol. 54 1723-42

Evans R D 1972 The Atomic Nucleus (New York: McGraw-Hill) pp 621-8

Fermi E 1934 Versuch einer Theorie der $\beta$-Strahlen. I, Z Physik 88 161-77

Fernández-Ramirez C, Moya de Guerra E, Udias A and Udias J M 2008 Properties of nucleon resonances by means of a genetic algorithm Phys. Rev. C 77065212

Fernández-Varea J M, Mayol R, Baró J and Salvat F 1993 On the theory and simulation of multiple elastic scattering of electrons Nucl. Instrum. Methods B 73 447-73

Fu L and Qi J 2010 A residual correction method for high-resolution PET reconstruction with application to on-the-fly Monte Carlo based model of positron range Med. Phys. 37 704-14

Gove N B and Martin M J 1971 Log-f tables for beta decay Nucl. Data Tables 10205

Haber S F, Derenzo S E and Uber D 1990 Application of mathematical removal of positron range blurring in positron emission tomography IEEE Trans. Nucl. Sci. 37 1293-9

Harpen M D 2004 Positronium: review of symmetry, conserved quantities and decay for the radiological physicist Med. Phys. 31 57-61

Harrison R L, Kaplan M S, Vannoy S D and Lewellen T K 1999 Positron range and coincidence non-collinearity in SimSET IEEE Nucl. Sci. Symp. Conf. Rec. pp 1265-8

Herzog H, Iida H, Weirich C, Tellmann L, Kaffanke J, Spellerberg S, Caldeira L, Kops E R and Shah N J 2010 Influence from high and ultra-high magnetic field on positron range measured with a 9.4TMR-BrainPET IEEE Nucl. Sci. Symp. Conf. Rec. pp 3410-3

Hirayama H, Namito Y, Bielajew A F, Wilderman S J and Nelson W R 2005 The EGS5 code System SLAC-R-730 and KEK Report 2005-8 (Stanford, CA: Stanford Linear Accelerator Center)

Hoffend J, Mier W, Schuhmacher J, Schmidt K, Dimitrakopoulou-Strauss A, Strauss L G, Eisenhut M, Kinscherf R and Haberkorn U 2005 Gallium-68-DOTA-albumin as a PET blood-pool marker: experimental evaluation in vivo Nucl. Med. Biol. 32 287-92

Hoffman E J, Phelps M E, Mullani N A, Higgins S C and Ter-Pogossian M M 1976 Design and performance characteristics of a whole-body positron transaxial tomograph J. Nucl. Med. 17 493-502 PMID: 1262967

ICRP 1975 Report of the Task Group on Reference Man ICRP Publication 23 (Oxford: Pergamon) 
ICRU 1989 Tissue Substitutes in Radiation Dosimetry and Measurement ICRU Report No 44 (Bethesda, MD: ICRU)

Jødal L, Le Loirec C and Champion C 2012 Positron range in PET imaging: an alternative approach for assessing and correcting the blurring Phys. Med. Biol. 57 3931-43

Katz L and Penfold A S 1952 Range-energy relations for electrons and the determination of beta-ray end-point energies by absorption Rev. Mod. Phys. 24 28-44

Kraus R, Delso G and Ziegler S I 2012 Simulation study of tissue-specific positron range correction for the new biograph mMR whole-body PET/MR system IEEE Trans. Nucl. Sci. 59 1900-9

Lehnert W, Gregoire M C, Reilhac A and Meikle S R 2011 Analytical positron range modelling in heterogeneous media for PET Monte Carlo simulation Phys. Med. Biol. 56 3313-35

Le Loirec C and Champion C 2007a Track structure simulation for positron emitters of medical interest: part I. The case of the allowed decay isotopes Nucl. Instrum. Methods Phys. Res. A 582 644-53

Le Loirec C and Champion C 2007b Track structure simulation for positron emitters of physical interest: part II. The case of the radiometals Nucl. Instrum. Methods Phys. Res. A 582 654-64

Le Loirec C and Champion C 2007c Track structure simulation for positron emitters of physical interest: part III. The case of the non-standard radionuclides Nucl. Instrum. Methods Phys. Res. A 582 665-72

Levin C S and Hoffman E J 1999 Calculation of positron range and its effect on the fundamental limit of positron emission tomography system spatial resolution Phys. Med. Biol. 44 781-99

Liljequist D 1987 Critical path length for the similarity of elastic multiple scattering processes J. Appl. Phys. 62 333-41

Muñoz A, Pérez J M, García G and Blanco F 2005 An approach to Monte Carlo simulation of low-energy electron and photon interactions in air Nucl. Instrum. Methods A 536 176-89

National Nuclear Data Center, Brookhaven National Laboratory 2011 www.nndc.bnl.gov/

Palmer M R and Brownell G L 1992 Annihilation density distribution calculations for medically important positron emitters IEEE Trans. Med. Imaging 11 373-8

Palmer M R, Zhu X and Parker J A 2005 Modeling and simulation of positron range effects for high resolution PET imaging IEEE Trans. Nucl. Sci. 52 1391-5

Panettieri V, Wennberg B, Gagliardi G, Duch M A, Ginjaume M and Lax I 2007 SBRT of lung tumours: Monte Carlo simulation with PENELOPE of dose distributions including respiratory motion and comparison with different treatment planning systems Phys. Med. Biol. 52 4265-81

Partridge M, Spinelli A, Ryder W and Hindorf C 2006 The effect of beta(+) energy on performance of a small animal PET camera Nucl. Instrum. Methods Phys. Res. A 568 933-6

Phelps M E, Hoffman E J and Huang S 1975 Effects of positron range on spatial resolution J. Nucl. Med. 16 649-52 PMID: 1151485

Poularikas A D (ed) 2000 The Transforms and Applications Handbook (Boca Raton, FL: CRC Press)

Rapisarda E, Bettinardi V, Thielemans K and Gilardi M C 2010 Image-based point spread function implementation in a fully 3D OSEM reconstruction algorithm for PET Phys. Med. Biol. 55 4131-51

Salvat F and Fernández-Varea J M 1992 Semiempirical cross sections for the simulation of the energy loss of electrons and positrons in matter Nucl. Instrum. Methods B 63 255-69

Salvat F, Fernández-Varea J M and Sempau J 2008 PENELOPE-2008_a code system for Monte Carlo simulation of electron and photon transport NEA No. 6416, Organisation For Economic Co-Operation And Development

Sánchez-Crespo A, Andreo P and Laarson S A 2004 Positron flight in human tissues and its influence on PET image spatial resolution Eur. J. Nucl. Med. Mol. Imaging 31 44-51

Sempau J and Andreo P 2006 Configuration of the electron transport algorithm of PENELOPE to simulate ion chambers Phys. Med. Biol. 51 3533-48

Soultanidis G, Karakatsanis N, Nikiforidis G and Loudos G 2011 Study of the effect of magnetic field in positron range using GATE simulation toolkit J. Phys.: Conf. Ser. 317012021

Westbrook C I, Gidley D W, Conti R S and Rich A 1989 Precision measurement of the orthopositronium vacuum decay rate using the gas technique Phys. Rev. A 405489

Wirrwar A, Vosberg H, Herzog H, Halling H, Weber S and Muller-Gartner H W 19974.5 tesla magnetic field reduces range of high-energy positrons-potential implications for positron emission tomography IEEE Trans. Nucl. Sci. 44 184-9

Wu C S and Moskowski S A 1966 Beta Decay (New York: Interscience)

X-5 Monte Carlo Team 2003 MCNP - a general Monte Carlo N-particle transport code, version 5 Report LA-UR03-1987 (Los Alamos, NM: Los Alamos National Laboratory)

Yoshinaga $\mathrm{K}$ et al 2006 What is the prognostic value of myocardial perfusion imaging using rubidium-82 positron emission tomography? J. Am. College Cardiol. 48 1029-39 